\title{
Protected Catalyst Growth of Graphene and Carbon Nanotubes
}

Muhammad Ahmad ${ }^{1}$, José V. Anguita ${ }^{1}$, C. Ducati ${ }^{2}$, J. David Carey ${ }^{1,3}$ and S. Ravi P. Silva*1,3

${ }^{1}$ Advanced Technology Institute, University of Surrey, Guildford, Surrey, GU2 7XH, United Kingdom

${ }^{2}$ Department of Materials Science \& Metallurgy, University of Cambridge, 27 Charles Babbage road, CB3 0FS Cambridge, UK

${ }^{3}$ Department of Electrical and Electronic Engineering, University of Surrey, Guildford, Surrey, GU2 7XH, United Kingdom

*Corresponding Author. Tel: $+\underline{44(0) 1483689825 .}$ Email: $\underline{\text { s.silva@ } \text { surrey.ac.uk }}$ 


\begin{abstract}
Some of the key challenges in the applications of graphene and carbon nanotubes are associated with their poor attachment to the substrate and poisoning of the catalyst by environmental contamination prior to the growth phase. Here we report a 'protected catalyst' technique which not only overcomes these challenges but also provides a new material production route compatible with many applications of carbon nanomaterials. The breakthrough technique involves capping the catalyst with a protective layer of a suitable material (examples include TiN, Cr, Ta etc.) which protects the catalyst from environmental contaminants such as oxidation, etchant attack, etc., whilst allows carbon supply to the catalyst for the CVD growth of desired nanomaterial. A thin Fe catalyst layer remained protected due to the capping layer in the $\mathrm{CF}_{4}$ based reactive-ion-etching of $\mathrm{SiO}_{2}$. We show that the carbon nanostructures grown using this technique exhibit significantly improved adhesion to the substrate in sonication bath tests. We demonstrate the fabrication of 3D structures and CNT based vias in a buried catalyst arrangement using the protected catalyst technique. The technique also allows better control over various growth parameters such as number of graphene layers, growth rate, morphology, and structural quality.
\end{abstract}




\section{Introduction}

Graphene and carbon nanotubes (CNTs), owing to their unique properties that include atomically thin stable structure, excellent mechanical properties, tunable electrical properties, high thermal conductivity, high corrosion resistance, high surface area to volume ratio (graphene), high aspect ratio (CNTs) have become highly sought nanomaterials for many potential applications in electronics, [1, 2] aerospace,[1] automobiles, paints and coatings, [1, 2] battery electrodes[1] and corrosion resistant barrier layers.[3, 4] For applications that need precise and accurately 'made-to-measure' nanostructures, chemical vapor deposition (CVD) is the preferred production method of these carbon nanomaterials.[5-7] Typical CVD production strategies involve the growth on a metal catalyst such as $\mathrm{Fe}, \mathrm{Ni}, \mathrm{Co}$, at high temperatures (600 $-1100^{\circ} \mathrm{C}$ ) in a hydrocarbon gas environment after performing a catalyst pre-heat treatment in a reducing gas (e.g. $\mathrm{H}_{2}$ ) environment.[5] For $\mathrm{CNTs}$, generally an oxide support layer (e.g. $\mathrm{SiO}_{2}$, $\mathrm{Al}_{2} \mathrm{O}_{3}$ ) under the catalyst is often employed; growth on conductive support layers is less common and remains challenging. $[5,8,9]$

Despite their excellent properties and a high application scope, there remain technological and processing challenges which hinder exploitation of these carbon nanomaterials to full potential. A significant difficulty of using in-situ CVD grown CNTs and graphene in many applications is their poor attachment to the substrate.[10] This poor attachment not only poses reliability issues, but also results in poor electrical and thermal contact with the underlying surface. A further issue is the catalyst contamination through oxidation, which mainly occurs when the sample after catalyst deposition under high vacuum conditions is exposed to air and transferred to the CVD chamber before the growth commences.[11, 12] Such contamination and formation of metal-(sub)oxides affect the growth and increase contact resistance at the underlying metal interface.[13, 14] In integrated circuit

\footnotetext{
*Corresponding Author. Tel: +44 (0)1483 689825. Email: s.silva@ surrey.ac.uk
} 
interconnects, the high current density requirement, and filling of reliable void-free high aspect ratio vias (vertical interconnect access) and TSVs (through silicon vias) using conventional top-down deposition techniques have been stated as amongst the foremost challenges for future interconnect technology.[15] CNTs, being able to grow from the bottom of a high aspect ratio via and owing to their excellent mechanical and electrical properties can ensure the realization of high current carrying void-free reliable vias. However, the deposition of the catalyst at the bottom of a high aspect ratio via remains challenging.[16] Here, we present a revolutionary 'protected-catalyst' technique which not only overcomes the aforementioned problems but may also open new application avenues and provide a significant breakthrough for many applications of graphene and CNTs.

\subsection{Protected Catalyst Technique}

Unlike the general scheme, the protected catalyst technique involves capping the catalyst by a thin protective layer (PrL) prior to the growth of graphene or CNTs as illustrated in Figure 1(a, b). The PrL protects the catalyst from oxidation, etchant attack and other contamination whilst allows carbon diffusion through it for the growth of the carbon nanomaterial. Importantly, the technique is applicable for both electrically conductive and non-conductive substrates. As the catalyst remains protected, the technique may be used to enhance the shelf life of growth-ready samples and their portability before initiating the growth phase. The technique offers an improved adhesion of the grown nanomaterials with the substrate, allows CNT based via fabrication in a buried catalyst scheme, can be used to produce 3D structures and provides control to tailor various properties of materials to be grown.

A range of materials such as $\mathrm{Ti}, \mathrm{TiN}, \mathrm{Ta}, \mathrm{TaN}, \mathrm{Au}, \mathrm{Ag}, \mathrm{Pd}, \mathrm{Cr}$ and polyimide are found here to act as a PrL. The choice and thickness of the material as PrL determines its functionality and the resulting properties of to be grown carbon nanomaterials. For example, a film of TiN is stable and resistant to some etchants, so a PrL of TiN can be useful in protecting the catalyst 
from oxidation and etchant attack, and to improve the adhesion of the nanomaterials, as depicted in Figure 1c. A PrL of high conductivity material such as Au, Ag, Pd etc., can be used for where high electrical conductivity is required and a PrL of polyimide can be configured such that it lifts-up with the growth of CNTs to form 3D structures, as illustrated in Figure 1d. Protection of the catalyst from etchants allows the fabrication of vias in a buried catalyst scheme, where all CNTs originate from the bottom (Figure 1e), in contrast with the general scheme where some CNTs originate from the sidewalls of vias (Figure 1f) due to catalyst deposition on the sidewalls.[17] Growth of graphene in protected catalyst scheme is illustrated in Figure 1g.
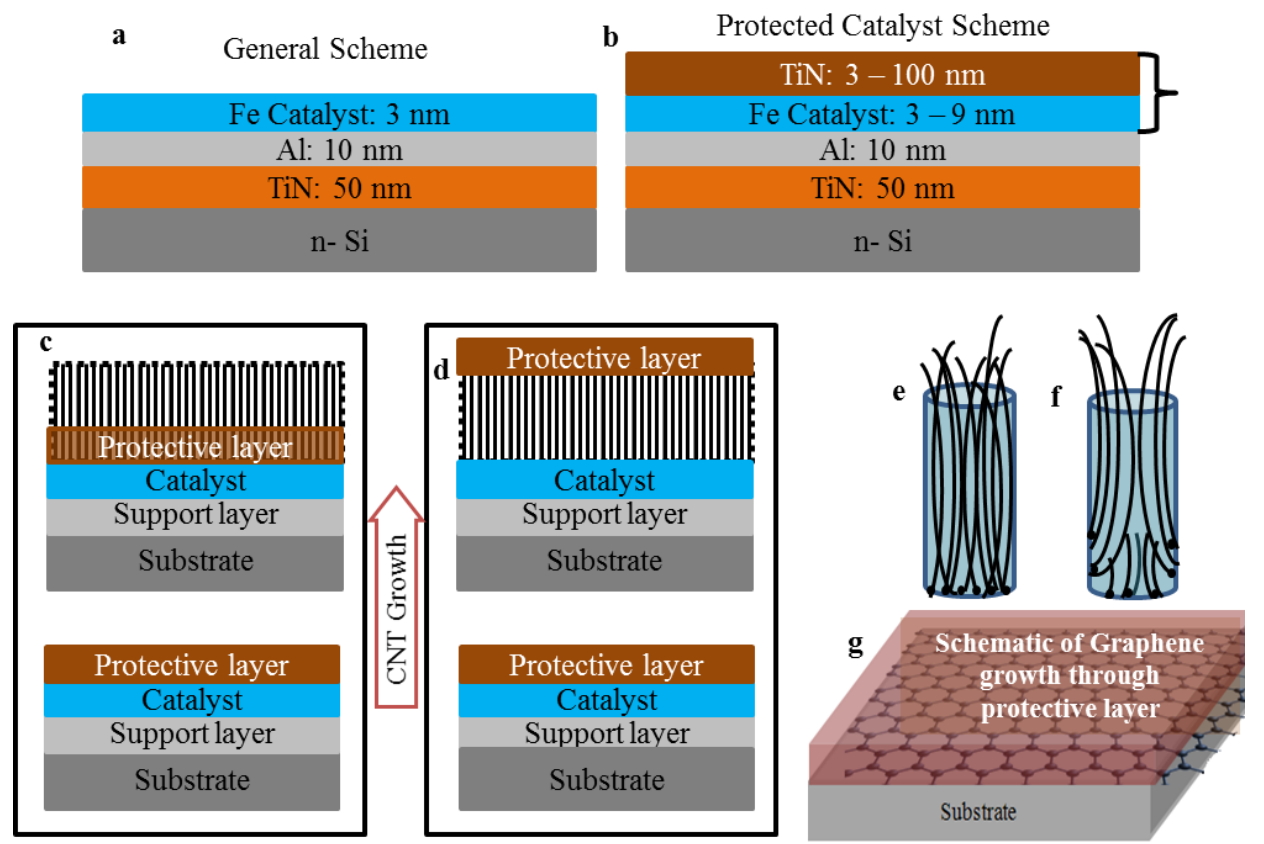

Figure 1. (a) General scheme where catalyst ( $\mathrm{Fe}$ ) is exposed and (b) protected catalyst scheme where a protective layer (PrL) of TiN protects the catalyst. (c) The scheme where the PrL protects the catalyst from environmental poisoning and etchant attack, and improves the adhesion of the carbon nanomaterials. (d) Lifting up of the PrL with the growth of the carbon nanomaterials to form 3D structures. (e) Illustration where all CNTs are growing from the bottom of a via in the protected catalyst scheme, whereas (f) some CNTs growing from sidewalls in the general scheme. (g) Schematic of graphene growth using a PrL. 


\section{Results and Discussion}

\subsection{Growth of CNTs using TiN as a Protective layer}

CNT growth in the protected catalyst scheme is thoroughly examined using TiN as a PrL on $\mathrm{Si} / \mathrm{TiN}(50 \mathrm{~nm}) / \mathrm{Al}(10 \mathrm{~nm}) / \mathrm{Fe}(3-9 \mathrm{~nm})$ structures. TiN is known for its good barrier properties, and high chemical and thermal stability, we utilized it to protect the catalyst from environmental contamination, etchant attack $\left(\mathrm{CF}_{4}\right.$ plasma based $\left.\mathrm{RIE}\right)$ and to promote the adhesion of carbon nanomaterials.[18-20] Schematics of the layer structures for both general and protected catalyst schemes are shown in Figure $1(\mathrm{a}, \mathrm{b})$. In order to demonstrate effectiveness of TiN PrL to protect the catalyst from oxidation and thus enhancing the shelf life of growth-ready samples, we performed XPS analysis on more than a year old samples containing $\mathrm{Al}(10 \mathrm{~nm}) / \mathrm{Fe}(3 \mathrm{~nm})$ layer stack with $\operatorname{TiN} \operatorname{PrL}(7.0 \mathrm{~nm})$ and without the $\operatorname{PrL}$ (Figure 2). The asymmetric shape of the peak at $706.98 \mathrm{eV}$ for the sample with TiN PrL confirms that the Fe catalyst remained in metallic form and has been protected effectively from oxidation. On the other hand, the peak has shifted to higher binding energies $(711.05 \mathrm{eV})$ for the sample without the PrL, indicating oxidation of the Fe catalyst.

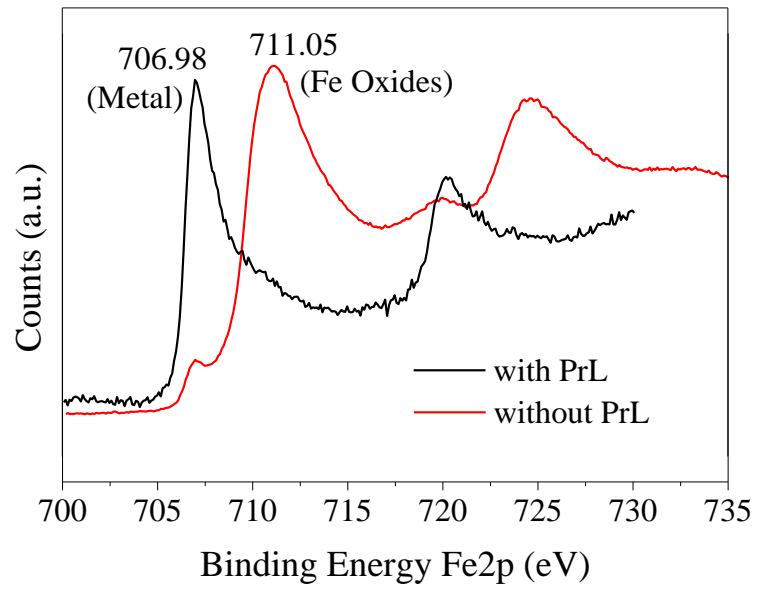

Figure 2. XPS spectra of a year old samples showing protection of the Fe catalyst from oxidation with the TiN PrL. 
For CNT growth, thickness of the Fe catalyst is selected variously to be $3.0 \mathrm{~nm}, 6.0 \mathrm{~nm}$ and $9.0 \mathrm{~nm}$ and that of the PrLs (TiN) to be $3.5 \mathrm{~nm}, 7.0 \mathrm{~nm}$ and $17 \mathrm{~nm}$. Growth is conducted in the substrate bulk temperature range of $375-440^{\circ} \mathrm{C}$ in a photo-thermal CVD (PTCVD) system for 5 minutes. PTCVD is an efficient growth method for carbon nanomaterials that relies on high power optical lamps for rapid energy delivery to the catalyst, while bulk of the substrate is held at lower temperatures (details given in Method).[7, 21] Figure 3 shows representative SEM images of the CNTs grown using $3.0 \mathrm{~nm}$ Fe catalyst with the PrL, whereas Figure S1 of the Supporting Information (SI) shows the corresponding images without the PrL. Vertically aligned and dense $\left(\sim 10^{9} \mathrm{~cm}^{-2}\right.$ estimated by analysis of SEM images) CNTs grown with the PrL are observed at $375^{\circ} \mathrm{C}$. The growth rate of the CNTs grown without the PrL at this temperature is $\sim 5.3 \mu \mathrm{m} / \mathrm{min}$, whereas it decreases from 2 to $1 \mu \mathrm{m} / \mathrm{min}$ as the thickness of the TiN PrL increases from 3.5 to $17 \mathrm{~nm}$, which indicates that the carbon supply to the catalyst can be controlled by the thickness of the PrL. As the temperature increases further, the vertical alignment and density of CNTs grown with the PrL is degraded.

Previous studies have shown that the thickness of the catalyst affects the growth of CNTs; a thin catalyst film is required to form small sized catalyst nanoparticles but too thin film may diffuse into the adjacent layers or alloy with the metal support at higher temperatures.[22, 23] In the current structure, the catalyst $(3.0 \mathrm{~nm} \mathrm{Fe})$ is sandwiched between the $\mathrm{Al}$ film at the bottom and TiN PrL at the top. In absence of the top TiN PrL, good CNT growth occurs in the whole temperature range as shown in Figure S1 of the SI; this suggests that the diffusion or alloying of the thin Fe film at higher temperatures occurs in the top TiN PrL and an increased Fe thickness may improve the CNT growth by providing access catalyst. To validate this hypothesis, we increased the thickness of the catalyst to $6.0 \mathrm{~nm}$ and $9.0 \mathrm{~nm}$, while keeping the PrL thicknesses the same. 

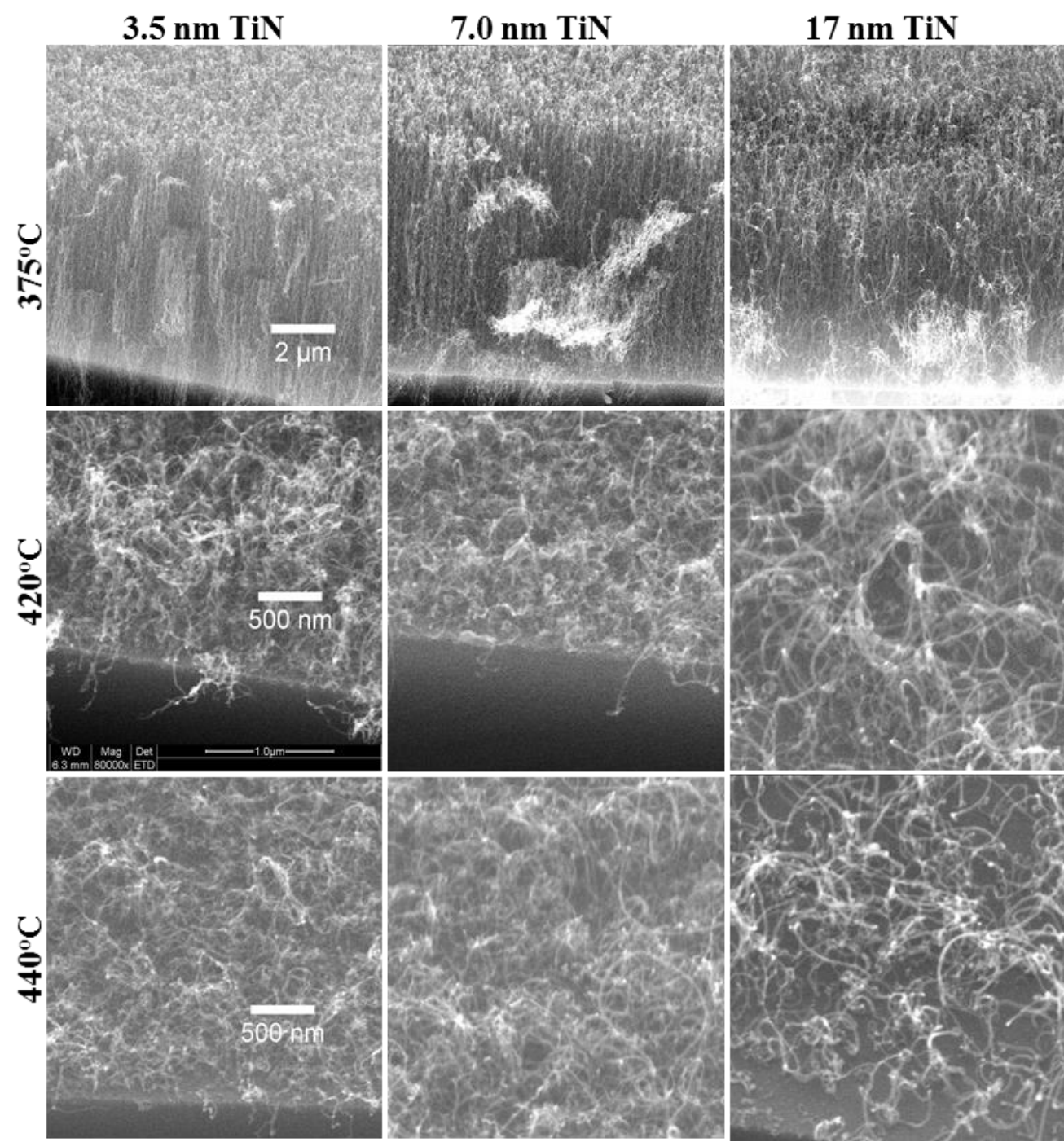

Figure 3. SEM images of the CNTs grown in the protected catalyst scheme for $3.0 \mathrm{~nm}$ Fe catalyst samples. The TiN PrL thicknesses are $3.5 \mathrm{~nm}, 7.0 \mathrm{~nm}$, and $17 \mathrm{~nm}$ and the growth substrate bulk temperature is $375-440^{\circ} \mathrm{C}$. Good vertically aligned CNT growth is observed at $375^{\circ} \mathrm{C}$, whereas tangled and sparse CNT growth occurred at higher temperatures. The scale bar in each of the rows are same.

The images of the CNTs grown using $6.0 \mathrm{~nm}$ and $9.0 \mathrm{~nm}$ Fe catalyst are shown in Figure $\mathrm{S} 2$ and Figure S3 of the SI, respectively. These figures show no or poor growth at $375^{\circ} \mathrm{C}$ but better growth at higher temperatures, in contrast with the CNTs grown using $3.0 \mathrm{~nm}$ Fe (Figure 3). So increasing the thickness of the catalyst improves the CNT growth at higher temperatures, whereas the catalyst film is too thick to form rightly sized active nanoparticles for the CNT growth at low temperatures.[24] CNT growth using a wide range of catalyst and PrL thicknesses shows the diversity of the protected catalyst technique which allows to tailor some 
of the properties of CNTs such as vertical alignment, height, density, etc. Some additional material segments can be seen on the CNTs grown using thicker catalyst films (Figure S2 and Figure S3 of the SI). We believe these segments are excess catalyst and not the TiN (PrL), since these are absent for thinner catalyst (3.0 nm Fe) for all thicknesses of TiN PrL (Figure 3). This suggests that the TiN film remains at the roots of the CNTs which improves their anchoring with the substrate, as will be discussed later.

Figure 4 contains the Raman spectra (514 nm laser) and analysis of the CNTs grown on $3.0 \mathrm{~nm}$ Fe with and without the TiN PrL in the temperature range of $375-440^{\circ} \mathrm{C}$. Well defined D peaks at $\sim 1340 \mathrm{~cm}^{-1}$, G peaks at $\sim 1580 \mathrm{~cm}^{-1}$ and $2 \mathrm{D}$ peaks at $\sim 2680 \mathrm{~cm}^{-1}$ are observed in all cases. The absence of the radial breathing mode indicates that the CNTs are multiwall. The D band in graphitic materials is attributed to the structural defects and explained by the double resonance process involving electron scattering from phonons and defects.[25] A strong D peak is usually observed for MWCNTs even for highly graphitized tubes, which is thought to be due to the turbostratic structure of the walls and defects at the ends of the tubes.[26] The G band corresponds to the Raman-active $\mathrm{E}_{2 \mathrm{~g}}$ vibration of neighboring $\mathrm{sp}^{2}$ carbon atoms and its intensity represents the degree of graphitization in the material.[27] The intensity ratio of the D and the G peaks $\left(\mathrm{I}_{\mathrm{D}} / \mathrm{I}_{\mathrm{G}}\right)$ is commonly used to compare the structural quality of the graphitic materials. The 2D band is the overtone of the $\mathrm{D}$ band but it is independent of the crystalline defects.[27] Since the D band is defect induced and the intensity of the 2D band increases with higher structural quality, their ratios $\left(\mathrm{I}_{\mathrm{D}} / \mathrm{I}_{2 \mathrm{D}}\right)$ can also be used as a metric of the structural quality. $[6$, 28] The sharper width of the peaks also represent high structural quality of graphitic materials.[29] 

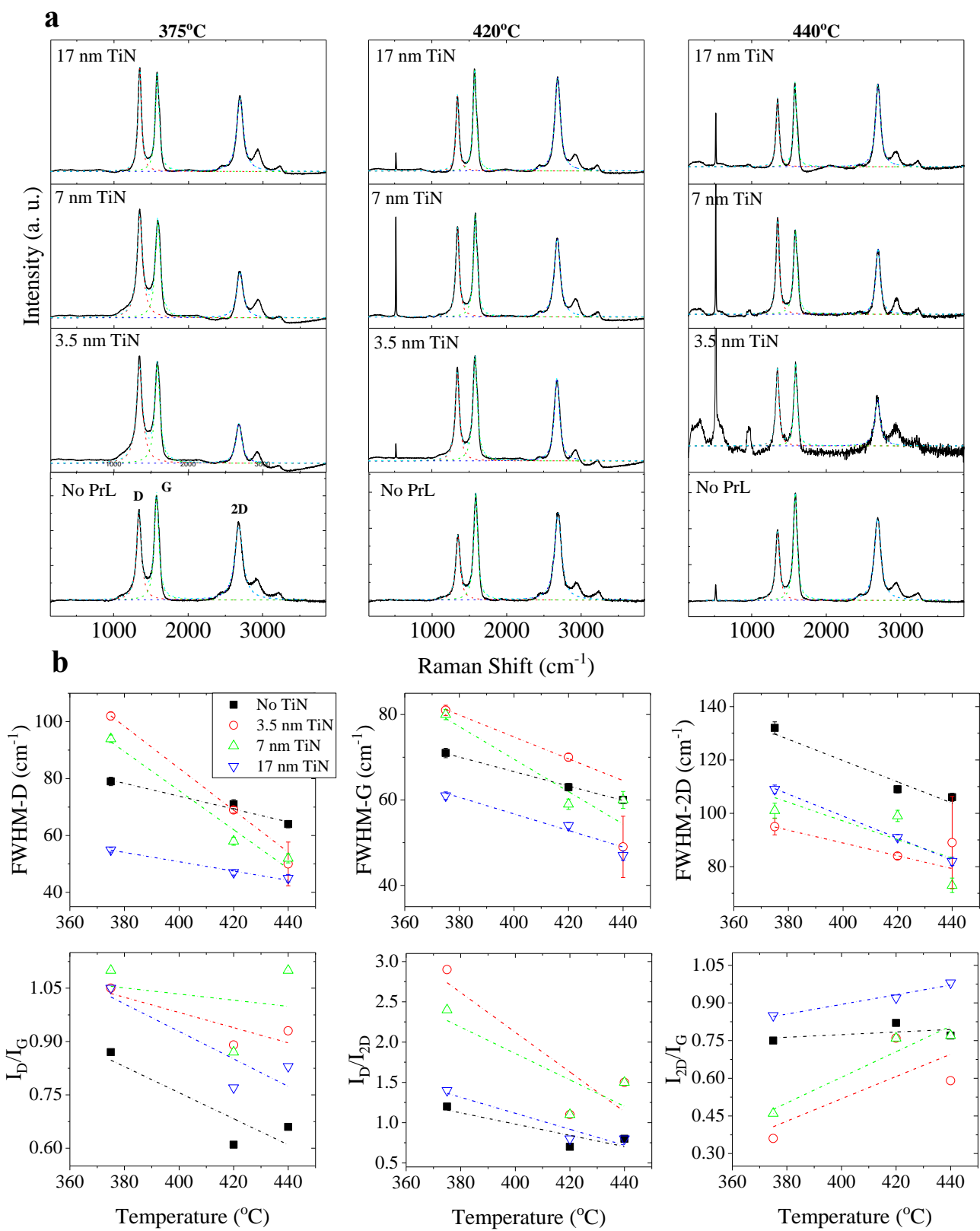

Figure 4. (a) Raman spectra of the CNTs grown using protected catalyst scheme for $3.0 \mathrm{~nm} \mathrm{Fe}$ in the substrate bulk temperature range of $375-440^{\circ} \mathrm{C}$. Well defined CNT characteristic D, G and 2D peaks at around $\sim 1340 \mathrm{~cm}^{-1}, \sim 1580 \mathrm{~cm}^{-1}$ and $\sim 2680 \mathrm{~cm}^{-1}$, respectively are observed. (b) FWHMs and various intensity peak ratios are plotted as a function of temperature for different thicknesses of TiN based PrL after fitting the peaks using a Lorentzian function.

The $\mathrm{D}, \mathrm{G}$ and 2D peaks are fitted using a single Lorentzian function, respectively. The full width at half maximum (FWHM) of the fitted peaks and their intensity ratios are plotted as a function of temperature in Figure 4(b). The FWHM of all peaks decreases with increasing growth temperature and the FWHM of the D and the G peaks also decreases with increasing 
the thickness of the TiN PrL. The FWHM for the samples with PrLs are narrower than those without PrL at higher growth temperatures. The $\mathrm{I}_{\mathrm{D}} / \mathrm{I}_{\mathrm{G}}$ and $\mathrm{I}_{\mathrm{D}} / \mathrm{I}_{2 \mathrm{D}}$ ratios also decrease for higher growth temperatures, indicating better structural quality. [30, 31]However, the overall quality of the CNTs, as judged by $\mathrm{I}_{\mathrm{D}} / \mathrm{I}_{\mathrm{G}}$ and $\mathrm{I}_{\mathrm{D}} / \mathrm{I}_{2 \mathrm{D}}$ ratios, is slightly lower $\left(\mathrm{I}_{\mathrm{D}} / \mathrm{I}_{\mathrm{G}}\right.$ of 0.77$)$ as compared with the CNTs grown without the $\operatorname{PrL}\left(\mathrm{I}_{\mathrm{D}} / \mathrm{I}_{\mathrm{G}}\right.$ of 0.61$)$. This is likely due to the additional energy required for the growth of CNTs to protrude through the TiN PrL. The measured $I_{D} / I_{G}$ ratio of 0.77 of the sample grown using the PrL is comparable to samples grown at a much higher temperature of $700^{\circ} \mathrm{C}$ without PrL. $[30,31]$ As the effective growth temperature is lower, any slight reduction in material quality represents a good trade off, along with additional benefits of the PrL. Lower structural quality $\left(\mathrm{I}_{\mathrm{D}} / \mathrm{I}_{\mathrm{G}}: 1.0-1.10\right)$ of the CNTs is achieved at low temperatures $\left(375^{\circ} \mathrm{C}\right)$ and improved quality $\left(\mathrm{I}_{\mathrm{D}} / \mathrm{I}_{\mathrm{G}}: 0.77-0.93\right)$ is achieved at higher temperatures, which is in agreement with previous studies.[31, 32]

The intensity of the $2 \mathrm{D}$ peak (Figure $4 \mathrm{a}$ ) and hence the $\mathrm{I}_{2 \mathrm{D}} / \mathrm{I}_{\mathrm{G}}$ (Figure $4 \mathrm{~b}$ ) ratio increases with increasing the thickness of the TiN based PrL. The 2D peak has been reported to be sensitive to the doping concentration in CNTs.[29, 33, 34] As the PrL contains TiN, there is a possibility of growing nitrogen doped CNTs, however, we discarded this because in the above mentioned reports, the $\mathrm{I}_{2 \mathrm{D}} / \mathrm{I}_{\mathrm{G}}$ ratio decreases and the $\mathrm{I}_{\mathrm{D}} / \mathrm{I}_{\mathrm{G}}$ ratio increases with increasing dopant concentration, $[33,34]$ whereas opposite behavior is observed in our case, which is an indicative of higher quality of the CNTs. $[6,21]$ TEM analysis presented below reveals that the diameter and number of walls of the CNTs become relatively uniform with increasing thickness of the TiN PrL, which may be the reason of achieving higher intensities of the G- and 2D-peaks, and their narrower FWHM. Therefore, we believe that increasing the thickness of the TiN PrL increases the structural quality of the CNTs with relatively narrower diameter distribution. This highlights the utility of the TiN based PrLs to protect the catalyst without greatly compromising the quality of carbon nanomaterials. 
Figure 5(a-c) shows TEM images and corresponding diameter distribution of the CNTs grown without PrL, with $3.5 \mathrm{~nm}$ TiN PrL and with $7.0 \mathrm{~nm}$ TiN PrL, respectively. The CNT diameter and number of walls extracted from TEM analysis are given in Table 1 . The tubes appear clean, well graphitized and exhibit a similar structural quality in all cases with presence of single (not shown), double (green arrow), triple (red arrows) and multi (blue arrows) wall structures. The diameter and shell distribution of the tubes tend to become narrow for the CNTs grown with the PrL, which indicates that the protected catalyst technique can be utilized to achieve CNTs with uniform diameter and number of shells.
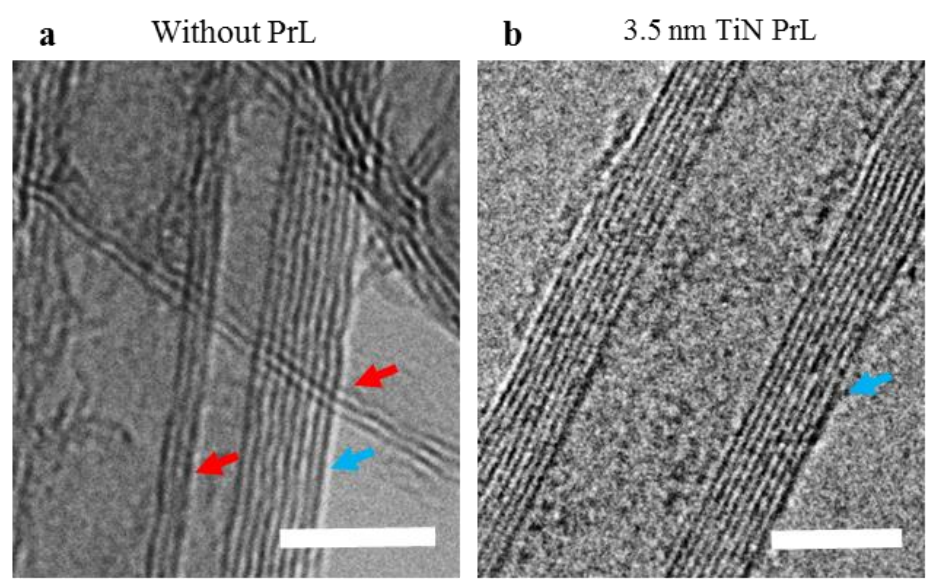

c $\quad 7.0 \mathrm{~nm} \mathrm{TiN} \mathrm{PrL}$
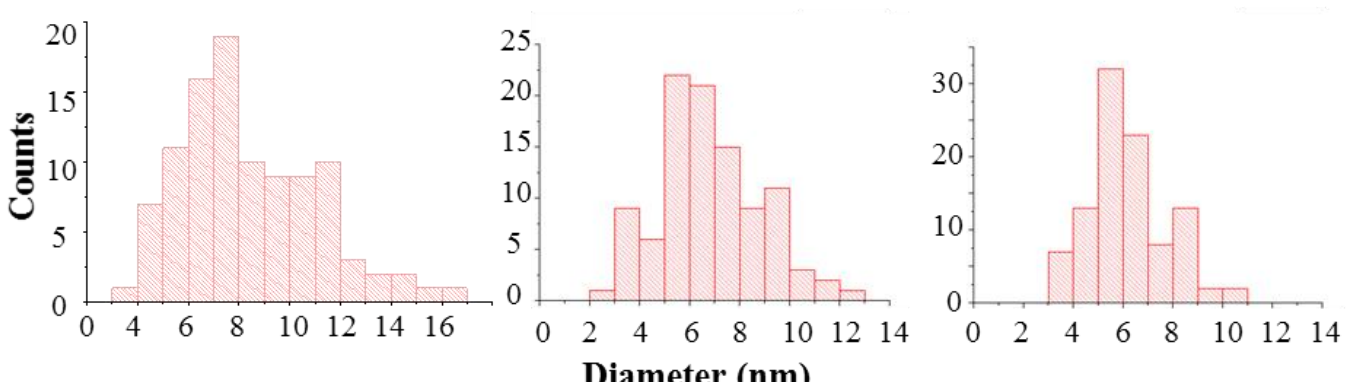

Figure 5. TEM images and corresponding diameter distribution analysis of the CNTs grown (a) without PrL, (b) with $3.5 \mathrm{~nm}$ TiN PrL and (c) with $7.0 \mathrm{~nm}$ TiN PrL. Green, red and blue arrows point to double walled, triple walled and multi walled CNTs. A relatively narrow CNT diameter distribution is observed for the CNTs grown with the PrL. Scale bar is $5 \mathrm{~nm}$. 
Table 1. CNT Diameter and number of walls extracted from TEM analysis.

\begin{tabular}{lccc}
\hline & Without PrL & 3.5 nm TiN PrL & 7.0 nm TiN PrL \\
\hline Av. Diameter (nm) & $8.4 \pm 2.7$ & $6.7 \pm 2$ & $6.2 \pm 1.5$ \\
$\begin{array}{l}\text { Dia. [Min - Max] } \\
\text { (nm) }\end{array}$ & $3.6-16.1$ & $2.9-12.3$ & $3.3-10.2$ \\
Walls (>50\% CNTs) & $2-6$ & $3-6$ & $3-6$ \\
Walls [Min - Max] & $1-12$ & $1-10$ & $1-7$ \\
\hline
\end{tabular}

\subsection{Adhesion of the Protected Catalyst Grown CNTs}

To compare the adhesion of the CNTs grown using the protected catalyst scheme and general scheme, sonication of the samples dipped into methanol vials is performed for one minute using $100 \%$ power of the sonicator (Ultrawave's Qi-100 ultrasonic cleaner).[10] Two CNT samples prepared using $3.5 \mathrm{~nm}$ and $7.0 \mathrm{~nm}$ TiN based PrL, and one sample prepared using general scheme were tested; SEM images are shown in Figure 6. All CNTs grown using general scheme were removed during the ultrasonic bath test. On the other hand, partial removal of CNTs occurred for $3.5 \mathrm{~nm}$ TiN PrL (Figure 6(c,d)) and nearly no removal observed for $7.0 \mathrm{~nm}$ TiN PrL (Figure 6(e, f)). This indicates improved adhesion of CNTs using the protected catalyst scheme, which is important for reliability and better electrical/thermal contact of the nanomaterials with the substrate. CNT densification in the solvent is also observed which diminished their vertical alignment.

The attachment of CNTs with the substrate improves by increasing the thickness of the PrL, as can be seen that $7.0 \mathrm{~nm}$ TiN PrL (Figure 6e) resulted in improved attachment as compared with that of $3.5 \mathrm{~nm}$ TiN PrL (Figure 6c). To further extend this, we used $100 \mathrm{~nm}$ TiN as $\operatorname{PrL}$ on lithographically patterned $\operatorname{Si} / \mathrm{TiN}(50 \mathrm{~nm}) / \mathrm{Al}(10 \mathrm{~nm}) / \mathrm{Fe}(6 \mathrm{~nm})$ squares $((100 \times 100$ $\mu \mathrm{m}^{2}$ ) and performed CNT growth at $440^{\circ} \mathrm{C}$ substrate bulk temperature using PTCVD. Figure 6(g, h) shows the SEM images where short CNTs are observed. Figure 6(g) shows the cross- 
section of cleaved sample where the CNT/PrL mat can be seen uncut, which indicates its high strength. Such robust CNT based structures are highly important and may be utilized for applications such as corrosion resistant battery electrodes, interconnects, thermal interface materials and supercapacitors.
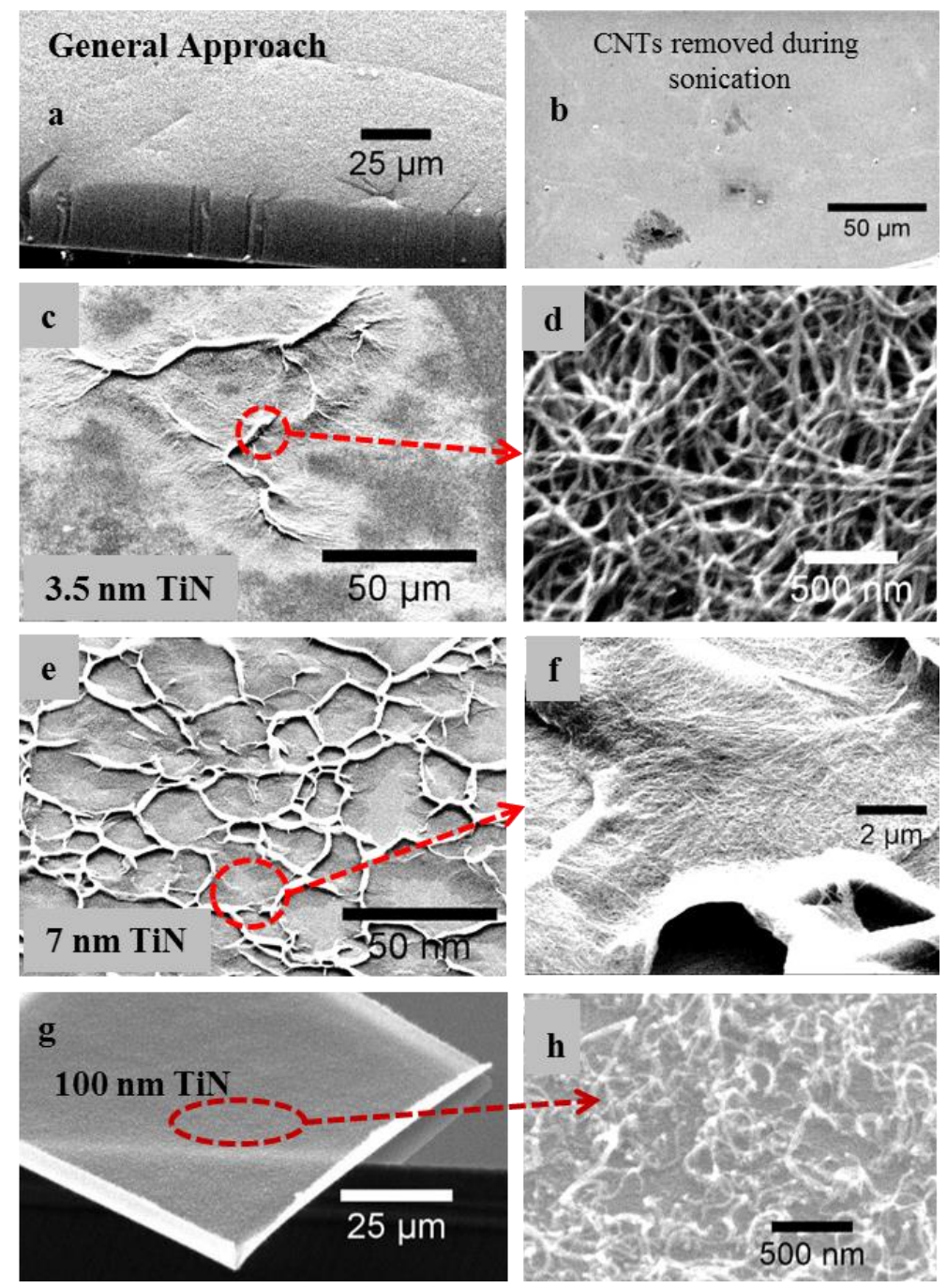

Figure 6. SEM images of CNTs for adhesion test. (a, b) CNTs grown using general scheme: (a) before sonication (b) after sonication where all CNTs are removed. (c, d) CNTs grown using $3.5 \mathrm{~nm}$ TiN PrL are partially removed, (e, f) CNTs grown using $7.0 \mathrm{~nm}$ TiN PrL are not removed, (g, h) CNTs grown using $100 \mathrm{~nm}$ TiN PrL.

The improved adhesion of the CNTs comes from the TiN based PrL which remains at the bottom of CNTs, as discussed earlier with respect to Figure 3, Figure S2 and Figure S3, where CNTs grown with $3.0 \mathrm{~nm}$ Fe were found clean from TiN contents for all selected thicknesses of the TiN PrL (Figure 3). This is further confirmed by EDX analysis performed 
along a line crossing a scratched area on a CNT grown sample with $7.0 \mathrm{~nm}$ TiN PrL (Figure 7a). In Figure 7b, no change in $\mathrm{Ti}$ (blue line) contents is observed along the scanned length which indicates that the TiN PrL remained at the bottom, otherwise reduced TiN contents were expected in the scratched area, as is the case for carbon (red line).
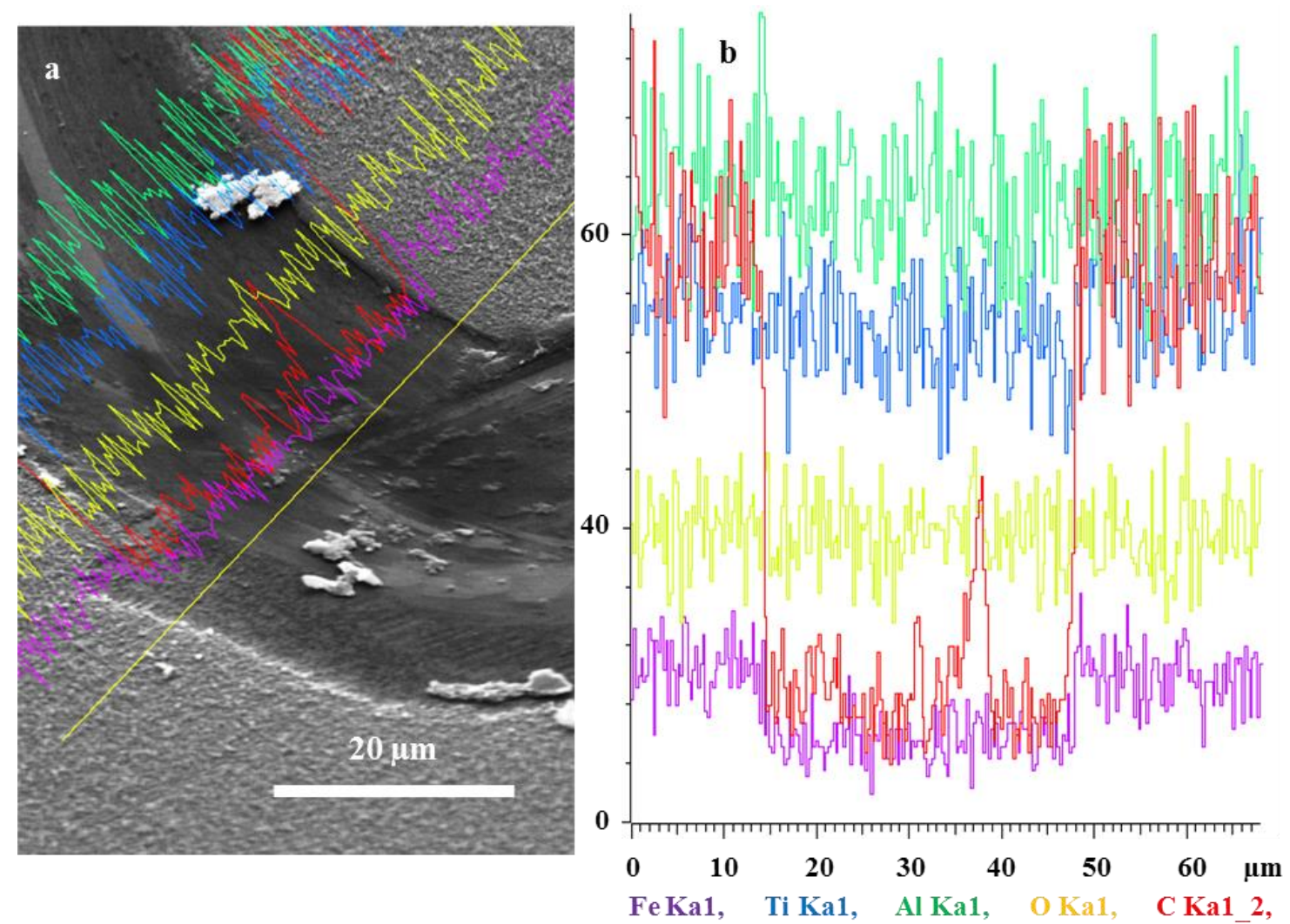

Figure 7. (a) SEM image of CNTs grown with $7.0 \mathrm{~nm}$ TiN PrL where EDX analysis is performed along the line crossing the scratched area. (b) EDX spectra showing no change in the Ti contents (blue line) indicating that TiN remained at the bottom of CNTs.

\subsection{Growth using Various Materials as PrL in PTCVD and Thermal CVD}

The protected catalyst scheme is not limited to only TiN based PrL, instead many other materials can also be used. For example, we demonstrate here the use of $\mathrm{Ti}, \mathrm{Cr}, \mathrm{Au}, \mathrm{Ag}, \mathrm{Pd}$ and $\mathrm{TaN}$ as a $\operatorname{PrL}(\sim 5.0 \mathrm{~nm})$ on a $\mathrm{Si} / \mathrm{TiN}(50 \mathrm{~nm}) / \mathrm{Al}(10 \mathrm{~nm}) / \mathrm{Fe}(4.5 \mathrm{~nm})$ structure. The CNT growth is conducted using PTCVD system at a substrate bulk temperatures of $420^{\circ} \mathrm{C}$, while keeping the other conditions identical to those described previously. Figure 8 shows SEM images where nearly vertically aligned CNTs with length in excess of a micron are observed 
for Ti and $\mathrm{Cr}$ based PrLs. Short and tangled CNTs for Ag and TaN and sparse CNTs for Au are seen. Short and thicker CNT bumps for Pd may have arisen by Pd wrapping around the CNTs during the growth due to enhanced wetting, which may be an interesting heterostructure for future research.
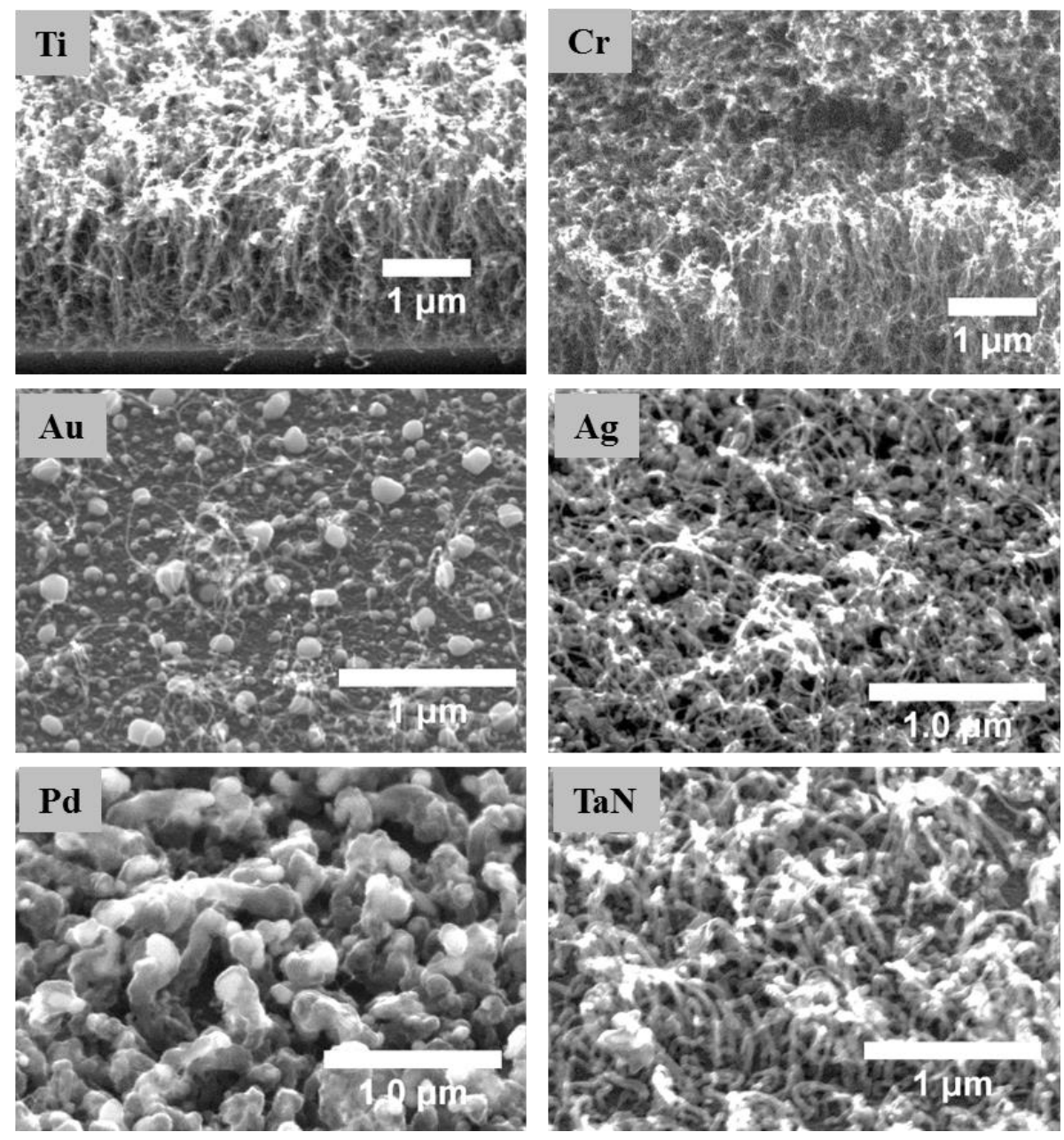

Figure 8. PTCVD grown CNTs in protected catalyst scheme using various materials as PrL. Vertically aligned CNTs of length $\sim 1 \mu \mathrm{m}$ for Ti and Cr based PrLs, short and tangled CNTs for Ag and TaN, Sparse CNTs for Au and thick short CNTs for Pd are observed.

There may be different routes of carbon supply to the catalyst for the growth of carbon nanomaterials depending on the selected material and thickness of the PrL. For transition metals (Ti, Ta, Cr, Au, Ag etc.) we believe that the supply depends on the carbon affinity in these materials, temperature, pressure and the thickness of PrL. For polymers and nitrides such as TiN and polyimide, voids and defects may also be the supply routes. For example, a TiN 
film contains voids and defects in its structure if the stoichiometry is not precisely controlled during the deposition process.[35] The presence of voids and defects in thin TiN film reduce its strength. At room temperature (or lower temperatures), the TiN film remains firm and uniform in protecting the catalyst effectively, however upon heating, the Fe film converts into nanoparticles which causes the TiN film to become porous and allow carbon diffusion through it, as illustrated in Figure 9(a).

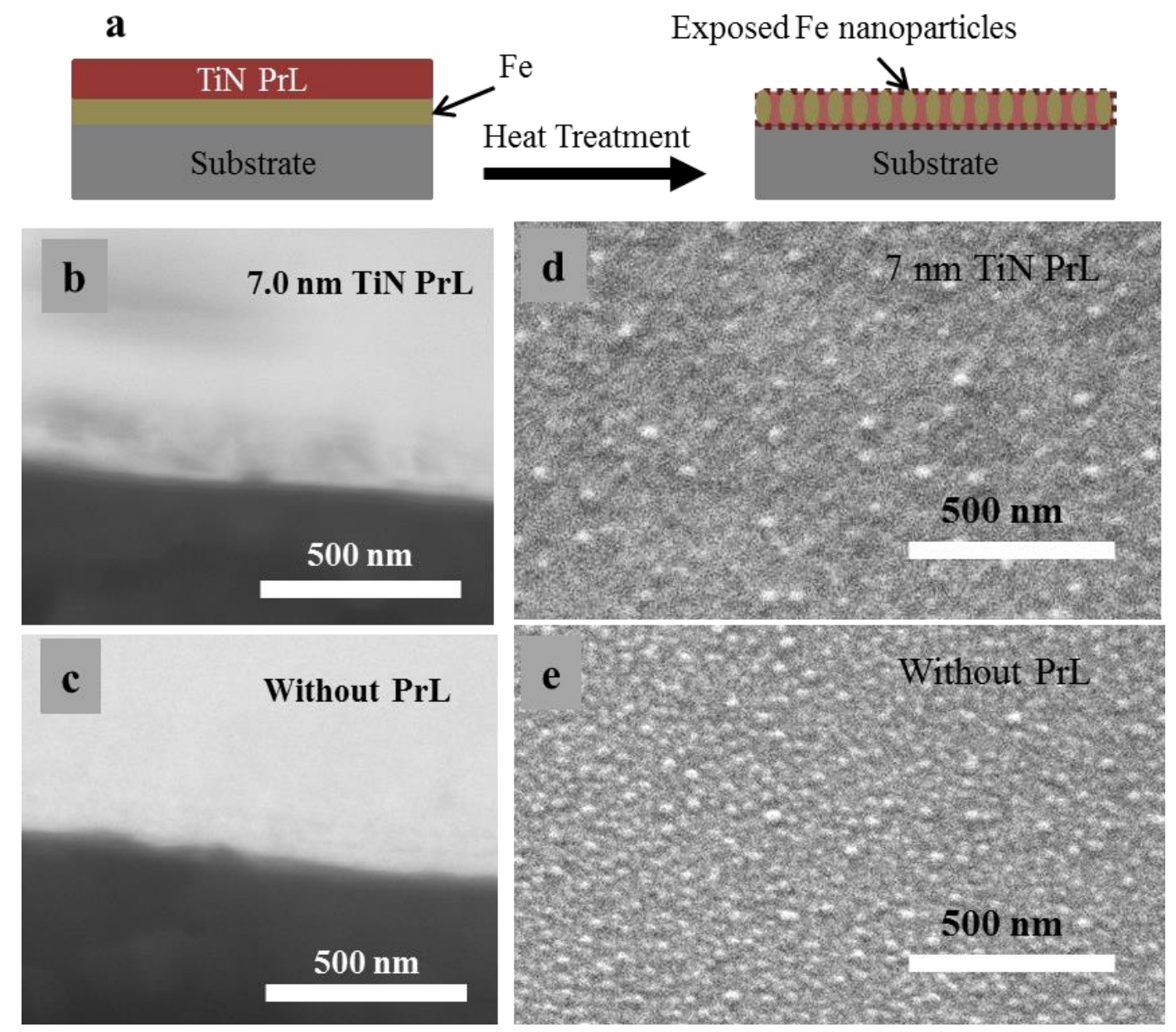

Figure 9. (a) Illustration of a carbon supply route in protected catalyst scheme. (b, c) Cross-section SEM images of the samples with and without PrL, respectively. (d, e) SEM images obtained after preheating the samples at $400^{\circ} \mathrm{C}$ with $7 \mathrm{~nm}$ TiN PrL showing exposed nanoparticles and without the PrL, respectively. 
In order to further support this view, we performed heat-treatment to samples with 7.0 $\mathrm{nm}$ TiN PrL and without the PrL at $400^{\circ} \mathrm{C}$. Figure 9(b-c) shows cross-section SEM images of the samples before annealing and Figure $9(\mathrm{~d}, \mathrm{e})$ shows top view of the samples after annealing where Fe nanoparticles can be seen. Bright catalyst particles can be seen exposed through the PrL in Figure 9(d), indicating formation of pin holes in the TiN PrL and protrusion of Fe nanoparticles. From Figure 8 it can be seen that each material gives its own distinctive CNT growth profile, which may be useful for various applications. For example, Ti and $\mathrm{Cr}$ based PrL can be used where protection of the catalyst from environmental contamination, corrosion, etchant attack and improved adhesion of the carbon nanomaterials is required, whereas $\mathrm{Au}, \mathrm{Ag}$ and Pd based PrLs are better suited for the applications where enhanced electrical conduction is needed.

The protected catalyst technique for the growth of carbon nanomaterials is not limited to the PTCVD, instead conventional CVD methods can also be utilized. As an example, we demonstrate CNT growth using protected catalyst scheme in a thermal CVD system (SNS Nanocat 5000). In this system, the stage where sample is mounted is heated with a resistive heating system which transfers the thermal energy to the catalyst for the growth. SEM images of the CNTs grown in the thermal CVD system are shown in Figure 10. Cr and Pd based PrLs produce dense CNTs, whereas Ti and Ta based PrLs result in relatively sparse CNTs. The diameter of CNTs for $\mathrm{Cr}$ and $\mathrm{Ta}$ is smaller as compared with that of $\mathrm{Ti}$ and $\mathrm{Pd}$, as observed by the SEM images. Au based PrL results in the production of CNT ropes and Ag in short CNT bumps. It is interesting to note difference in the PTCVD and thermal CVD grown CNTs (Figure 8 and Figure 10). For example, PTCVD grown CNTs using Ti as PrL are vertically aligned and dense, whereas the corresponding thermal CVD grown CNTs are relatively sparse. 

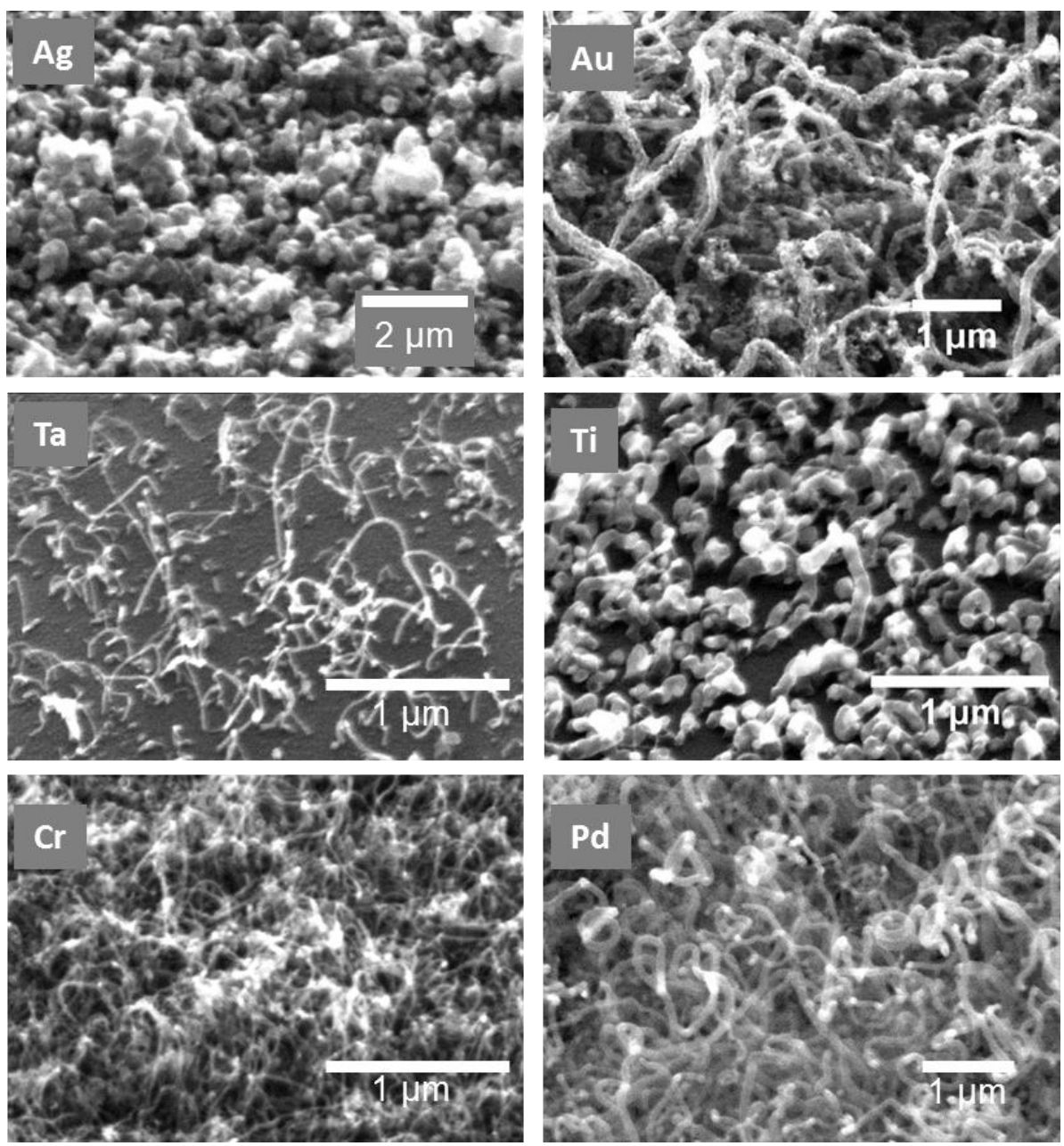

Figure 10. CNTs grown using thermal CVD with various materials as PrL. Cr and Pd based PrLs result in dense CNTs whereas Ta and Ti produce relatively sparse CNTs. Au results in CNT ropes and Ag in short CNT bumps.

The Raman spectra of the PTCVD and thermal CVD grown CNTs with various materials as PrL are shown in Figure 11. The spectra show distinct CNT characteristics including the G band, the D band and the 2D band. High structural quality of the PTCVD grown CNTs, as inferred from the lower $\mathrm{I}_{\mathrm{D}} / \mathrm{I}_{\mathrm{G}}$ values $(0.49-0.75)$, is achieved for TaN, Cr, Ti and Ag. The overall quality of the PTCVD grown CNTs is better when compared with the corresponding Raman spectra of the CNTs grown using thermal CVD, as inferred from lower $\mathrm{I}_{\mathrm{D}} / \mathrm{I}_{\mathrm{G}}$ values, enhanced 2D peaks and sharper Raman features. From above results, it is clear that the growth rate, morphology and structural quality of the CNTs can be tailored by the choice of the PrL 
material and its thickness, which is advantageous as it allows application specific customized CNT growth with tailored properties.
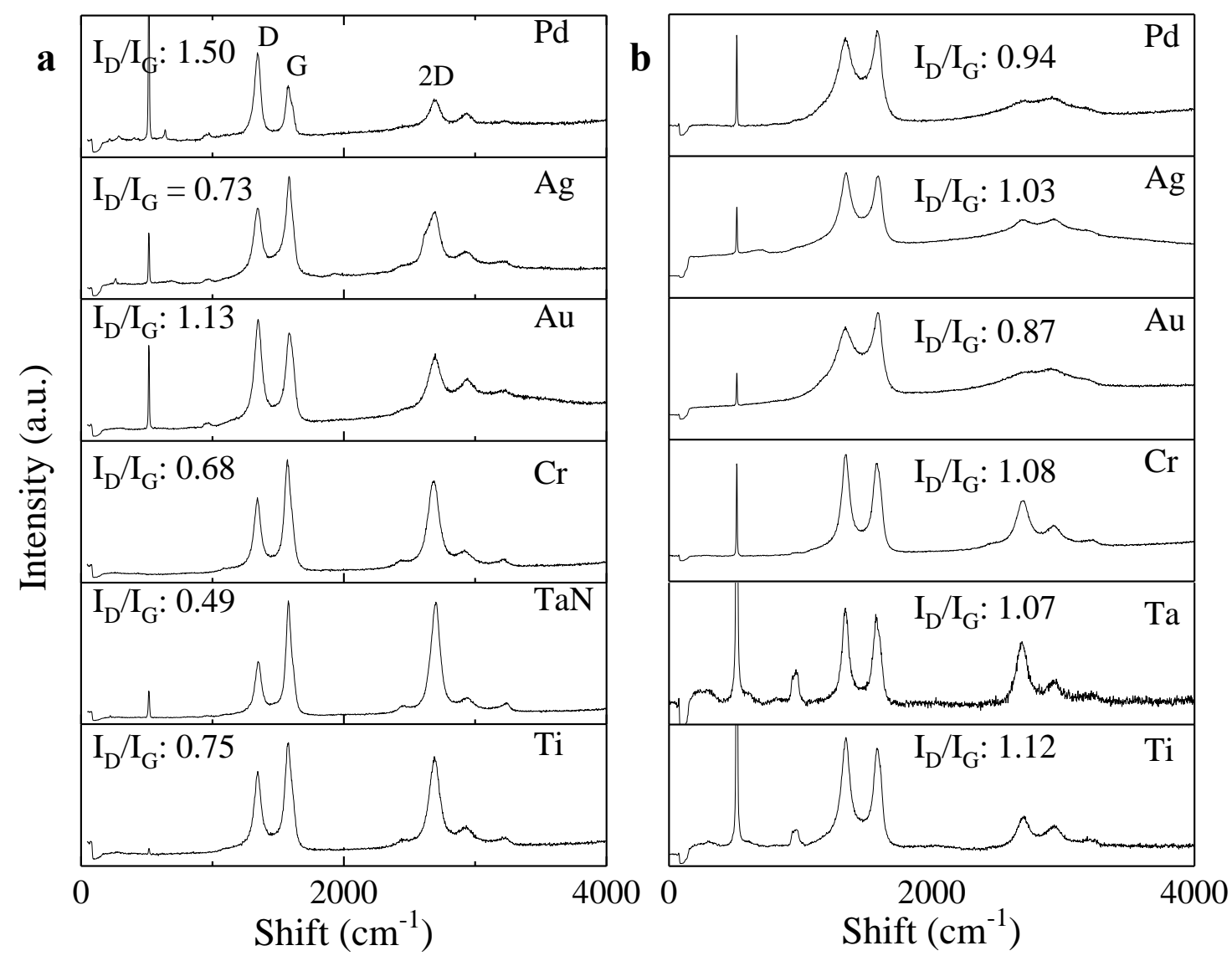

Figure 11. (a) Raman Spectra of the CNTs grown with different materials as PrL using: (a) PTCVD, (b) thermal CVD.

\subsection{Fabrication of CNT based Vias and 3D Structures}

The protected catalyst scheme can be used to fabricate reliable high aspect ratio CNT based vias. The process flow for the fabrication of vias both in the protected catalyst and general schemes are shown in Figure 12. In general scheme, the catalyst is deposited (step 3) after etching holes in a dielectric layer (step 2), which results in the side-wall deposition.[16, 36, 37] The sidewall deposition of the catalyst results in the growth of parasitic CNTs originating from the dielectric layer, which also hinders the growth of active CNTs growing from the bottom metal lines, as shown in Figure S4 of the SI. One approach to overcome this issue is to deposit the catalyst film before the deposition and subsequent etching of the dielectric layer.[17, 38] 
However, protecting the thin catalyst layer during the etching of the dielectric layer is highly challenging. We demonstrate here the use of protected catalyst technique to overcome this issue by protecting the catalyst during the etching step. Further detail of the via fabrication process is given in the Method. The catalyst remained protected due to the $7.0 \mathrm{~nm}$ TiN PrL during the reactive ion etching of the $\mathrm{SiO}_{2}$ layer which was performed using a mixture of $\mathrm{CF}_{4}$ and $\mathrm{Ar}$.

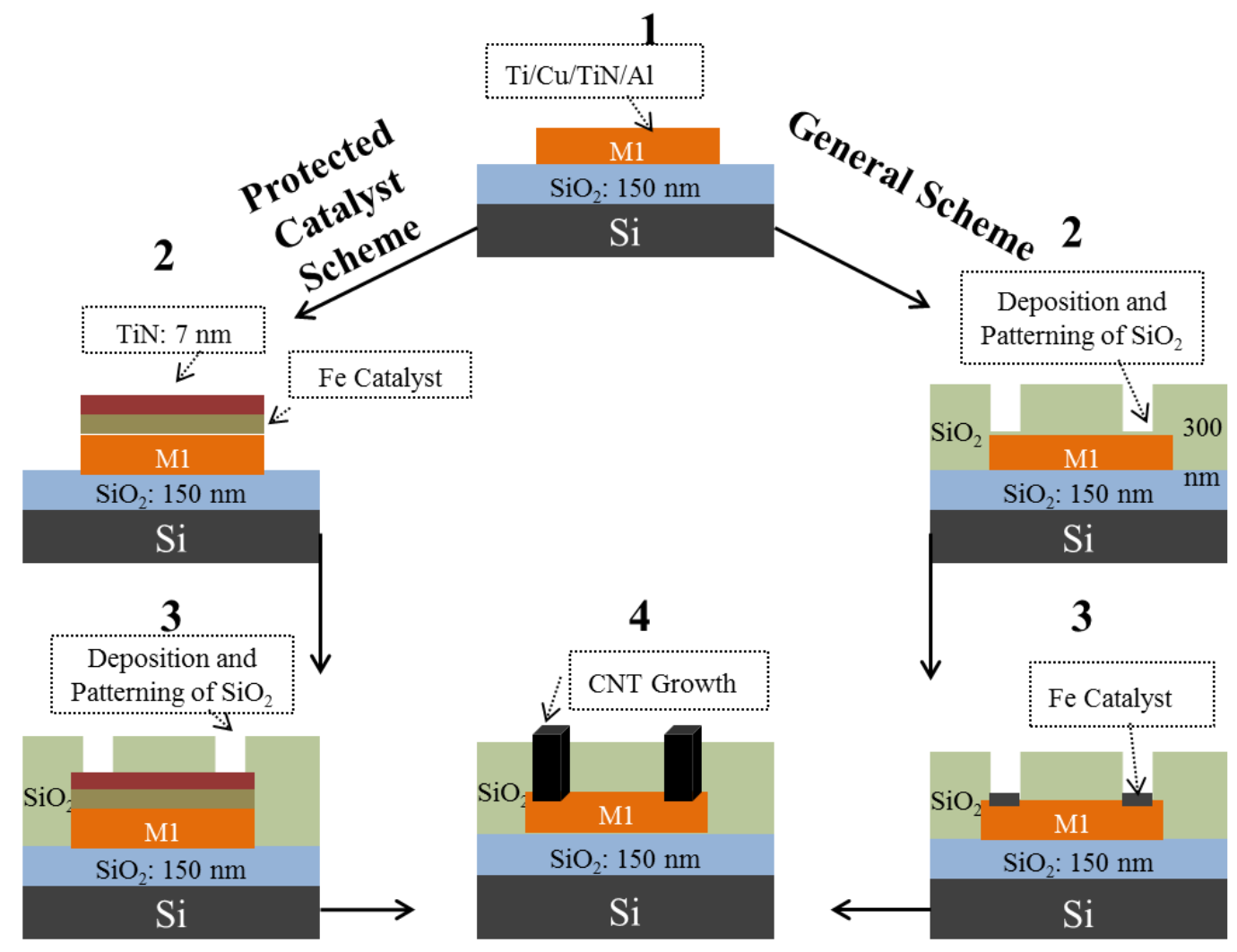

Figure 12. Schematic of the process flow for the fabrication of CNT based vias using the protected catalyst scheme and general scheme. In protected catalyst scheme, $3.0 \mathrm{~nm}$ Fe is capped with $7.0 \mathrm{~nm}$ TiN (step 2) and then $300 \mathrm{~nm} \mathrm{SiO}_{2}$ is deposited, patterned and etched (step 3). In general scheme, $\mathrm{Fe}$ (catalyst) is deposited (step 3) after the deposition, patterning and etching of $\mathrm{SiO}_{2}$ (step 2).

The PTCVD grown CNTs in $10 \times 10 \mu \mathrm{m}^{2}$ vias in a chain structure using protected catalyst technique are shown in Figure 13a, where a good vertically aligned and dense $\left(\sim 10^{9} \mathrm{~cm}^{-2}\right)$ growth of CNTs of length $5 \pm 1 \mu \mathrm{m}$ can be observed. In the protected catalyst scheme, it is evident that all CNTs originate from the bottom of the vias and have a good electrical contact as a result of improved attachment with the bottom metal layer. Figure $13 \mathrm{~b}$ shows the current- 
voltage characteristic of $100 \mathrm{CNT}$-based vias of $10 \times 10 \mu \mathrm{m}^{2}$ area fabricated through protected catalyst (solid line) and general (dashed line) schemes. Ohmic behavior is observed in both cases with a significant improvement in the calculated resistance $\sim 21 \Omega$ (resistivity: $\sim 4.2 \times 10^{-4}$ $\Omega m$ ) of a single CNT-based via fabricated using protected catalyst technique as compared with that of fabricated using general scheme $(\sim 280 \Omega)$. The improvement in the conductivity is attributed to the protection of the catalyst from oxidation, better electrical contact due to improved CNT attachment and increased number of CNTs available for conduction because of the inhibition of parasitic CNT growth from the sidewalls. The calculated resistivity of $4.2 \times 10^{-}$ ${ }^{4} \Omega \mathrm{m}$ for the CNT based vias using protected catalyst scheme is comparable with some of the best values reported in the literature.[7, 16, 39-41]. The protection of the thin catalyst film during the etching step and the improved conductivity of the CNT based vias provide evidence of TiN PrL role in protecting the catalyst from etchant attack and oxidation. The patterned growth using protected catalyst scheme is not limited to CNTs, instead it may be extended to combine graphene to act as horizontal interconnects.

In order to demonstrate 3D structures, $\sim 500 \mathrm{~nm}$ spin coated polyimide film (see Method for details) on $\mathrm{Si} / \mathrm{TiN}(50 \mathrm{~nm}) / \mathrm{Al}(10 \mathrm{~nm}) / \mathrm{Fe}(3 \mathrm{~nm})$ is used as a PrL. The CNT growth is performed in the PTCVD system at $400^{\circ} \mathrm{C}$ substrate temperature for 5 minutes. The SEM image is shown in Figure 13c, where the polyimide film can be observed at the top of the CNTs, indicating the lifting up of the polyimide film with the growth of CNTs and forming a 3D structure as depicted schematically in Figure $13 \mathrm{~d}$. The high thermal stability of the polyimide film (PI-5878G: $1 \% \mathrm{wt}$ loss at $560^{\circ} \mathrm{C}$ in air and decomposition temperature of $580^{\circ} \mathrm{C}$ ) allowed it to withstand the CNT growth temperatures at the top side of the samples. The factors which played a role in the lifting-up of the polyimide film include its structure comprising stable aromatic backbone network, its low mass density $(1.42 \mathrm{~g} / \mathrm{cm})$ and its high thermal stability. The growing CNTs would have acted as pillars to lift-up the polyimide film. Such 3D structure 
can be useful in revealing various topographical patterns and fewer steps in sandwiching CNTs between two metallic and/or insulating layers.
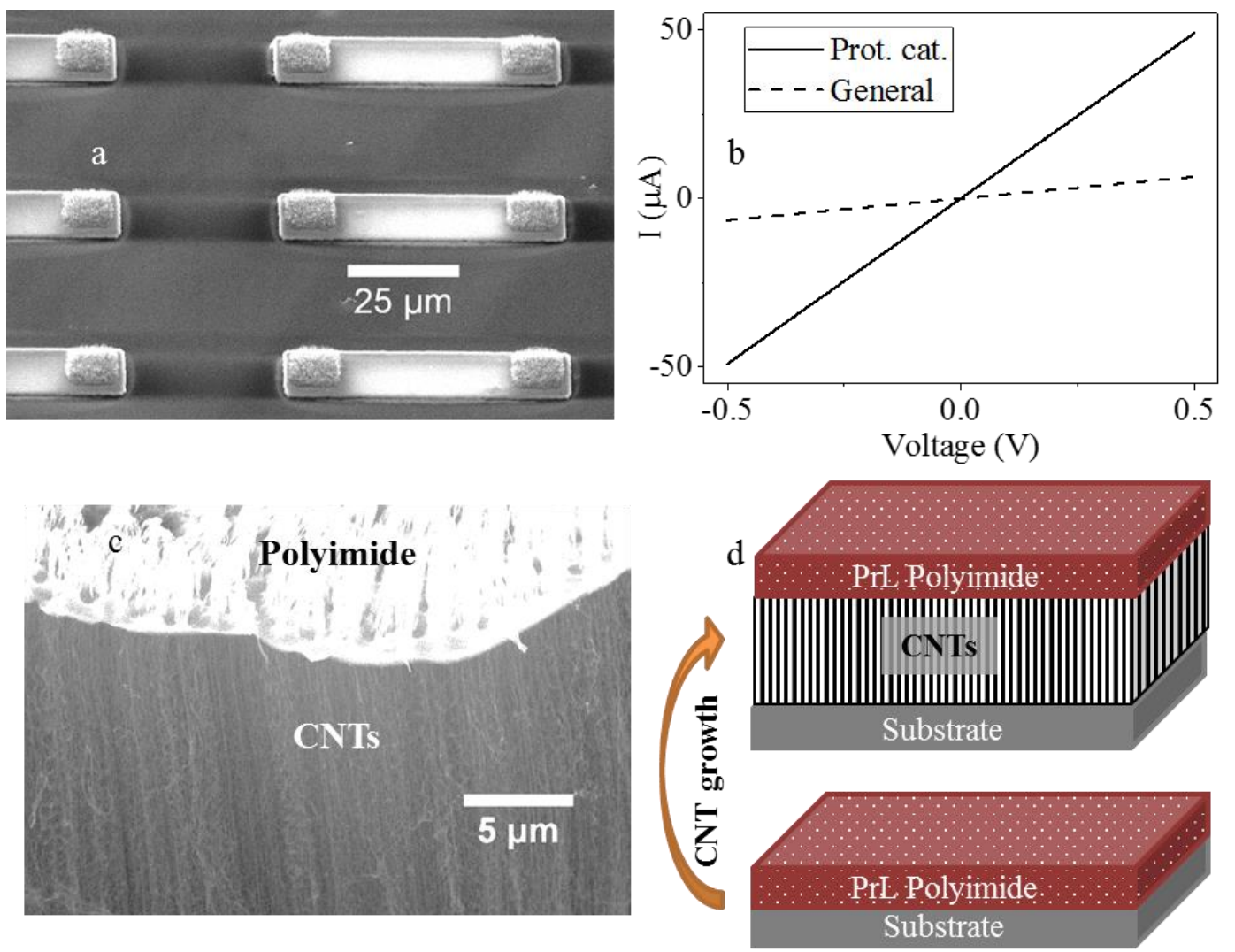

Figure 13. CNT based vias and 3D structures fabricated using the protected catalyst technique. (a) SEM micrograph of CNTs grown in vias using protected catalyst scheme. (b) Current-voltage plot of 100 CNT based vias in a chain structure showing higher conductivity for the vias fabricated using protected catalyst scheme (solid line). (c) SEM image showing the top PrL of polyimide ( $500 \mathrm{~nm})$ lifted up with the growth of CNTs, forming a 3D structure. (d) Schematic of 3D structure formation.

\section{Graphene Growth}

Growth of graphene using $7.0 \mathrm{~nm}$ TiN as PrL is demonstrated on $\mathrm{Si}$, Ni foil and steel sheets (1 mm thick). For Si, sputtered Fe (200 nm) and Ni (100 nm) are used as catalysts, whereas only Fe $(200 \mathrm{~nm})$ is used for growth on steel substrates. No separate deposition of catalyst is needed for growth on Ni foils. For comparison, graphene growth is also performed without the PrL. A schematic of the respective layer structures is shown in Figure S5 of the SI. Graphene growth is carried out for 5 minutes in the PTCVD system at a substrate bulk temperature of $450^{\circ} \mathrm{C}$. 
Further details about the growth conditions are given in Method. Figure $\mathbf{1 4}$ shows Raman spectra of as-grown graphene on various substrates.
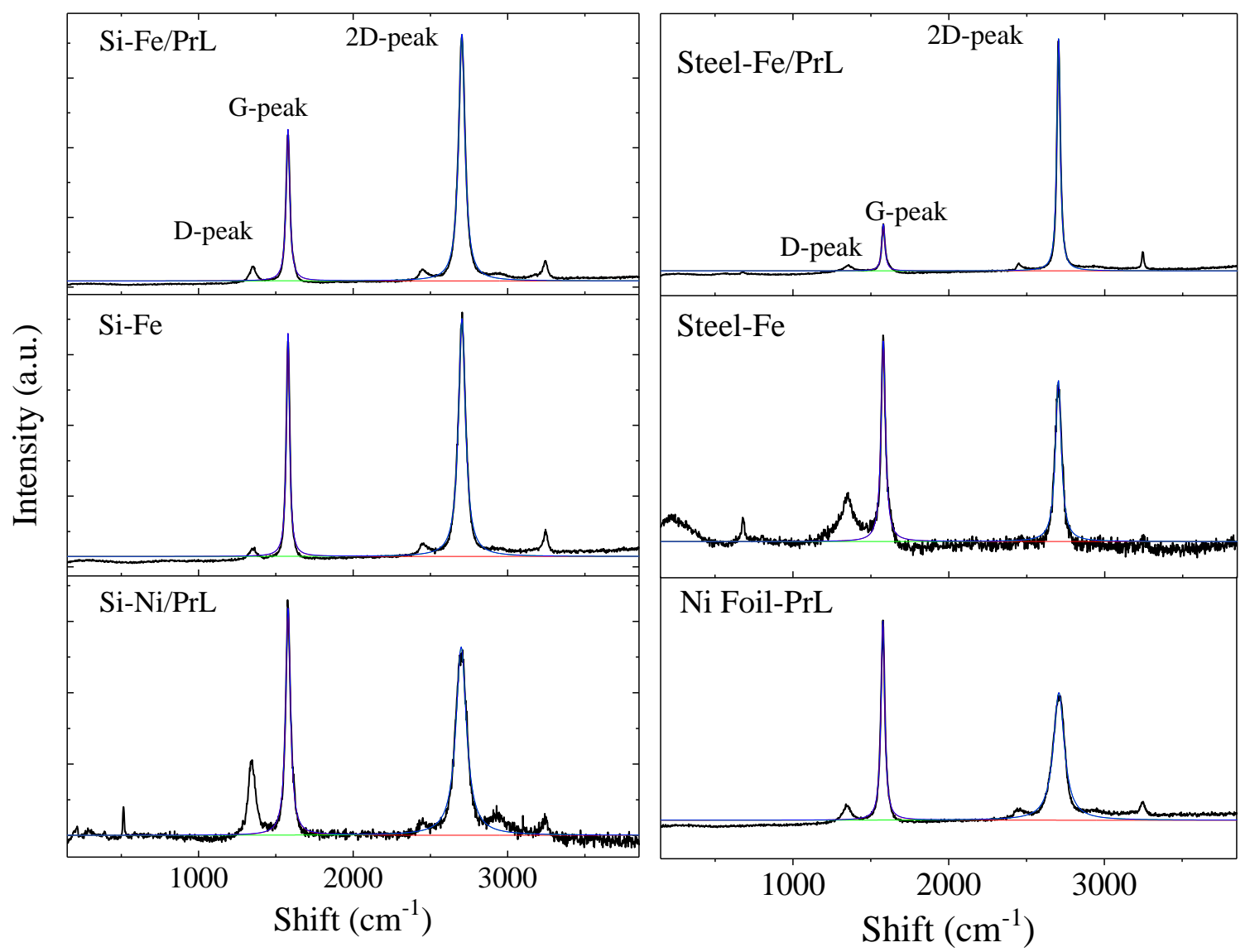

Figure 14. Raman spectra of graphene grown using protected catalyst technique on Ni foil, Si and Steel substrates. Fe or Ni are used as catalyst and $7.0 \mathrm{~nm}$ TiN as PrL. High quality growth of single layered, few layered and multi-layered graphene is achieved on various samples.

Well-defined first and second order Raman features are observed in all cases. The peaks are fitted using Lorentzian function and the values of $\mathrm{I}_{\mathrm{D}} / \mathrm{I}_{\mathrm{G}}, \mathrm{I}_{\mathrm{G}} / \mathrm{I}_{2 \mathrm{D}}$ and $\mathrm{FWHM}$ of the $\mathrm{G}$ and the 2D bands are given in Table 2 . The low $\mathrm{I}_{\mathrm{D}} / \mathrm{I}_{\mathrm{G}}$ values $(0.08-0.32)$ indicate high structural quality of the as-grown graphene. The shape and respective height of the 2D-peak with respect to the G-peak $\left(\mathrm{I}_{\mathrm{G}} / \mathrm{I}_{2 \mathrm{D}}\right)$ is associated with the interlayer coupling of electronic band structure and number of graphene layers, respectively.[42,43] The 2D-peak in a Raman spectrum of graphite is broader and has two components, whereas single-layer-graphene (SLG) shows a narrow single Lorentzian 2D-peak because of the absence of other graphene layers.[42] 
Figure S6 of the SI shows magnified 2D-peaks of the Raman spectra of steel samples with PrL and without PrL. The $\mathrm{I}_{\mathrm{G}} / \mathrm{I}_{2 \mathrm{D}}$ of 0.24 , sharp FWHM (full width half maximum) of 26 $\mathrm{cm}^{-1}$ and single Lorentzian shape of the peak in Figure S6(a) confirms the growth of singlelayer graphene on the steel substrate using protected catalyst scheme. On the other hand, the $\mathrm{I}_{\mathrm{G}} / \mathrm{I}_{2 \mathrm{D}}$ of 1.23 and FWHM of $48 \mathrm{~cm}^{-1}$ of the 2D-peak in Figure $\mathrm{S} 6(\mathrm{~b})$ indicates growth of few layered graphene (FLG) on the steel sample without PrL. The single Lorentzian shape of the peak indicates absence of interlayer coupling of electronic band structure, which is in agreement with literature reports and advantageous for maintaining intrinsic SLG properties in each layer of FLG.[21, 44]

Table 2. Values of $\mathrm{I}_{\mathrm{D}} / \mathrm{I}_{\mathrm{G}}, \mathrm{I}_{\mathrm{G}} / \mathrm{I}_{2 \mathrm{D}}$ and $\mathrm{FWHM}$ of the $\mathrm{G}$ and the $2 \mathrm{D}$ peaks of the Raman spectra.

\begin{tabular}{lcccc}
\hline Sample & $\mathbf{I}_{\mathbf{D}} / \mathbf{I}_{\mathbf{G}}$ & $\mathbf{I}_{\mathbf{G}} \mathbf{I}_{\mathbf{2 D}}$ & $\mathbf{F W H M ~ G}\left(\mathbf{c m}^{-\mathbf{1}}\right)$ & FWHM 2D $\left._{(\mathbf{c m}} \mathbf{- 1}^{\mathbf{1}}\right)$ \\
\hline Si-Ni/PrL & 0.32 & 1.3 & 38 & 82 \\
$\mathrm{Si}-\mathrm{Fe}$ & 0.08 & 0.89 & 25 & 55 \\
$\mathrm{Si}-\mathrm{Fe} / \mathrm{PrL}$ & 0.13 & 0.61 & 29 & 49 \\
$\mathrm{Ni}$ Foil/PrL & 0.13 & 1.56 & 29 & 85 \\
Steel-Fe & 0.24 & 1.23 & 34 & 45 \\
Steel-Fe/PrL & 0.26 & 0.24 & 24 & 26 \\
\hline
\end{tabular}

Similarly, the $\mathrm{I}_{\mathrm{G}} / \mathrm{I}_{2 \mathrm{D}}$ ratio of $1.56,1.30$, and 0.89 of Ni-foil/PrL, Si/Ni/PrL and $\mathrm{Si} / \mathrm{Fe}$ samples corresponds to multilayer graphene and that of $\mathrm{Si} / \mathrm{Fe} / \operatorname{PrL}\left(\mathrm{I}_{\mathrm{G}} / \mathrm{I}_{2 \mathrm{D}}: 0.61\right)$ corresponds to FLG. This implies that the PrL can be configured to control the number of graphene layers on a substrate. The graphene growth using protected catalyst scheme has high application potential in various areas such as flow-cell battery electrodes, solar cells, as corrosion resistant barrier layer on large area metal structures in the construction industry, etc. We believe that the demonstration of graphene growth on various substrates using protected catalyst scheme is highly important in view of research and industrial applications as it protects the catalyst and the grown material, improves adhesion and allows to control the number of layers of graphene. 


\section{Conclusion}

We demonstrate a unique 'protected catalyst' technique for the growth of carbon nanomaterials where a capping layer is used to protect the catalyst. The effect of catalyst (Fe) thickness on the CNT growth for various thicknesses of TiN based PrL is studied where thicker catalyst layers improve CNT growth at higher temperatures and thinner catalyst layers produce good quality CNTs at lower temperatures. CNTs of various characteristics (alignment, length, density, etc.) are achieved by varying the thickness and material of the PrL, both in PTCVD and thermal CVD methods. The protected catalyst scheme is also used to fabricate 3D structures and CNT-based vias which resulted in improved electrical conductivity. CNTs grown by the protected catalyst scheme are found more strongly attached with the substrate and the attachment strength increases with increasing thickness of the PrL. High quality single to multi-layer graphene growth using the protected catalyst scheme is also demonstrated. The protected catalyst scheme can provide significant technology for many application of the carbon nanomaterials as it simultaneously protects catalyst, improves the adhesion, allows dielectric etching while maintaining the catalyst intact and allows portability of growth ready catalyst all around the world.

\section{Method}

Schematic layer structures for general and protected catalyst scheme for CNT growth are shown in Figure 1(a, b), respectively. In protected catalyst scheme $50 \mathrm{~nm} \mathrm{TiN,} 10 \mathrm{~nm} \mathrm{Al}$, and $3.0-9.0 \mathrm{~nm}$ Fe (catalyst) films are sputter deposited on Si substrate followed by the deposition of $3.0-100 \mathrm{~nm}$ TiN film as a PrL. Apart from TiN, $5.0 \mathrm{~nm}$ film of various other materials including Ti, Ta, TaN, Pd, Ag, Au and polyimide are also investigated to act as a PrL. Graphene growth in protected catalyst scheme is conducted on Ni foil, Si and steel substrates. $200 \mathrm{~nm} \mathrm{Fe}$ or $100 \mathrm{~nm} \mathrm{Ni}$ film is sputter deposited on Si and steel substrates followed by the deposition of $7.0 \mathrm{~nm}$ TiN as a PrL, as shown in Figure S5 of the SI, whereas only $7.0 \mathrm{~nm}$ TiN as a PrL is 
deposited on Ni foil. All films on a sample are deposited sequentially without breaking the vacuum in an inert gas environment under high vacuum conditions using JLS-Design MPS 500 sputter kit.

Process flow for the fabrication of CNT-based vias using general and the protected catalyst scheme is shown in Figure 12. $150 \mathrm{~nm} \mathrm{SiO} 2$ film is sputtered on n-Si substrate followed by the deposition of the first metal layer (M1) which is a layer stack of Ti $(30 \mathrm{~nm})$, $\mathrm{Cu}(150 \mathrm{~nm})$, TiN $(50 \mathrm{~nm})$ and $\mathrm{Al}(10 \mathrm{~nm})$. In the general scheme, $3.0 \mathrm{~nm}$ Fe catalyst is deposited (step 3) after the deposition, patterning and etching (RIE) of $300 \mathrm{~nm} \mathrm{SiO}$ (step 2). On the other hand, in the protected catalyst scheme, $3.0 \mathrm{~nm} \mathrm{Fe}$ and $7.0 \mathrm{~nm}$ TiN PrLs are deposited (step 2) first and then deposition, patterning and etching of the $300 \mathrm{~nm} \mathrm{SiO} 2$ film (step 3) is performed. The RIE (reactive ion etching) of the $\mathrm{SiO}_{2}$ film is performed using a mixture of $\mathrm{CF}_{4}(30 \mathrm{sccm}) / \mathrm{Ar}(10 \mathrm{sccm})$ at $60 \mathrm{mTorr}$ pressure and 100 watt RF power. Growth of CNTs is performed using the PTCVD system at a substrate bulk temperature of $400^{\circ} \mathrm{C}$ (step 3). A $300 \mathrm{~nm} \mathrm{Al} \mathrm{film} \mathrm{is} \mathrm{sputter} \mathrm{deposited} \mathrm{on} \mathrm{CNT} \mathrm{based} \mathrm{vias,} \mathrm{patterned} \mathrm{and} \mathrm{etched} \mathrm{as} \mathrm{a} \mathrm{top}$ metal contact. Finally, electrical measurements of the CNT-based vias are performed.

To reveal 3D structures, $\sim 3.0 \mu \mathrm{m}$ polyimide (PI-5878G, purchased from HD MicroSystemsTM) as a PrL is spin-coated at $5000 \mathrm{rpm}$ on $\mathrm{Si} / \mathrm{TiN}(50 \mathrm{~nm}) / \mathrm{Al}(10 \mathrm{~nm}) / \mathrm{Fe}(3 \mathrm{~nm})$ structure. After the coating, soft baking of the film is performed on a hotplate at $110^{\circ} \mathrm{C}$ for 5 minutes and then the film is cured in two steps in $\mathrm{N}_{2}$ environment; first at $200^{\circ} \mathrm{C}$ for 30 minutes and then at $350^{\circ} \mathrm{C}$ for 1 hour. After this, $\mathrm{O}_{2}$ plasma etching $\left(30 \mathrm{sccm} \mathrm{O}_{2}\right.$ at $60 \mathrm{mTorr}$ and 100 watt $\mathrm{rf}$ power) is performed to reduce the thickness of the film to $\sim 500 \mathrm{~nm}$, using JLS-Design RIE80.

The growth of carbon nanotubes/graphene is mainly performed in a Photo-thermal CVD (PTCVD) system.[7, 21, 28, 45] However, some samples are also prepared using a conventional stage-heating CVD system (SNS 5001 Nanocat) to validate the versatility of the 
protected catalyst scheme. Details about PTCVD (SNS 1000n) can be found elsewhere.[21, 28, 46, 47] Briefly, in PTCVD, 8 lamps (1 kW each) in the optical-head deliver optical energy through a quartz window directly on the catalyst at the top surface of a sample in the reaction chamber, while the sample itself is placed on a water-cooled chuck to keep its bulk temperature low. In this arrangement, the top surface of the sample reaches the temperature between $600-$ $750^{\circ} \mathrm{C}$ depending on the electrical power supplied to the lamps, whereas the corresponding bulk temperature remains between $375-450^{\circ} \mathrm{C}$. A plot showing the top surface temperature of a sample measured by a thermocouple corresponding to the bulk temperature measured by a pyrometer at the backside of a sample, is given in Figure S7 of the SI.

For the PTCVD growth of CNTs/graphene, samples are preheated in $100 \mathrm{sccm}$ (standard cubic centimeter per minute) $\mathrm{H}_{2}$ flow for $10 \mathrm{~min}$ at 2.0 Torr pressure in a substrate bulk temperature range of $375-450^{\circ} \mathrm{C}$. After this, CNT/graphene growth is carried out using 10 sccm of $\mathrm{C}_{2} \mathrm{H}_{2}$ for 1.0 to $10 \mathrm{~min}$. In thermal CVD (SNS 5001 Nanocat), CNT growth is carried out at $700^{\circ} \mathrm{C}$ for 10 minutes using a mixture of $\mathrm{C}_{2} \mathrm{H}_{2} / \mathrm{H}_{2}(25 / 200 \mathrm{sccm})$ at 10 Torr pressure. Samples were pre-heated for 10 minutes in $\mathrm{H}_{2}$ environment prior to start the growth. Characterization of the samples is performed using an FEI quanta 200 scanning electron microscope (SEM), FEI Tecnai F20 microscope operated at $200 \mathrm{kV}$ and equipped with a Gatan OneView camera (TEM), ThermoFisher Scientific Instruments (East Grinstead,UK) K-Alpha + spectrometer for X-ray photoelectron spectroscopy (XPS), Renishaw Systems 2000 Raman Spectroscope (514 nm laser wavelength) and Keithley 4200 analyzer for electrical measurements. 


\section{Author Contributions}

S. Ravi P. Silva (SRPS) and Muhammad Ahmad (MA) proposed the project and defined the procedure. MA performed the experiments \& characterization. Caterina Ducati performed TEM imaging and analysis. MA and SRPS conducted the analysis. MA wrote the manuscript. SRPS and J. David Carey supervised the work. All authors revised, discussed and commented on the manuscript.

\section{Competing Financial Interests statement}

MA and SRPS are co-inventors on a patent application (WO2015189575 A1) owned by University of Surrey, which discloses the protected catalyst technique for the synthesis of carbon nanotubes/graphene and their use in various applications.

\section{Additional information}

Supplementary Information is provided with this paper.

\section{Acknowledgements}

The authors acknowledge financial support from EPSRC (EP/L02263x/1 and EP/R025304/1) in the conduct of this work. Access to TEM equipment was provided under the Henry Royce Institute's grants EP/P024947/1 and EP/R00661X/1. Authors would also like to thank Dr Steven Hinder of University of Surrey for his support in obtaining and analyzing XPS data. 


\section{References}

[1] M.F.L. De Volder, S.H. Tawfick, R.H. Baughman, A.J. Hart, Carbon Nanotubes: Present and Future Commercial Applications, Science (2013) 339(6119) 535.

[2] K.S. Novoselov, V.I. Falko, L. Colombo, P.R. Gellert, M.G. Schwab, K. Kim, A Roadmap for Graphene, Nature (2012) 490(7419) 192-200.

[3] J.M. Schnorr, T.M. Swager, Emerging Applications of Carbon Nanotubes, Chemistry of Materials (2011) 23(3) 646-657.

[4] Y. Zhang, L. Zhang, C. Zhou, Review of Chemical Vapor Deposition of Graphene and Related Applications, Accounts of Chemical Research (2013) 46(10) 2329-2339.

[5] M. Kumar, Y. Ando, Chemical Vapor Deposition of Carbon Nanotubes: A Review on Growth Mechanism and Mass Production, Journal of Nanoscience and Nanotechnology (2010) 10(6) 3739-3758.

[6] J. Campos-Delgado, Y.A. Kim, T. Hayashi, A. Morelos-Gomez, M. Hofmann, H. Muramatsu, et al., Thermal stability studies of CVD-grown graphene nanoribbons: Defect annealing and loop formation, Chemical Physics Letters (2009) 469(1-3) 177-182.

[7] M. Ahmad, J.V. Anguita, V. Stolojan, T. Corless, J.-S. Chen, J.D. Carey, et al., High Quality Carbon Nanotubes on Conductive Substrates Grown at Low Temperatures, Advanced Functional Materials (2015) 25(28) 4419-4429.

[8] B.C. Bayer, M. Fouquet, R. Blume, C.T. Wirth, R.S. Weatherup, K. Ogata, et al., CoCatalytic Solid-State Reduction Applied to Carbon Nanotube Growth, Journal of Physical Chemistry C (2012) 116(1) 1107-1113.

[9] K. Banerjee, L. Hong, N. Srivastava, Current Status and Future Perspectives of Carbon Nanotube Interconnects, 8th IEEE Conference on Nanotechnology, Arlington, TX, USA, 2008, pp. 432-436.

[10] H.-C. Su, C.-H. Chen, Y.-C. Chen, D.-J. Yao, H. Chen, Y.-C. Chang, et al., Improving the adhesion of carbon nanotubes to a substrate using microwave treatment, Carbon (2010) 48(3) 805-812.

[11] K.Y. Lee, S.I. Honda, M. Katayama, T. Miyake, K. Himuro, K. Oura, et al., Vertically aligned growth of carbon nanotubes with long length and high density, Journal of Vacuum Science \& Technology B (2005) 23(4) 1450-1453.

[12] B.C. Bayer, S. Hofmann, C. Castellarin-Cudia, R. Blume, C. Baehtz, S. Esconjauregui, et al., Support-Catalyst-Gas Interactions during Carbon Nanotube Growth on Metallic Ta Films, Journal of Physical Chemistry C (2011) 115(11) 4359-4369.

[13] R. Smith, F. Brown, A. R. Chester, Growth of Tall Vertical Carbon Nanotube Forests using Al-Fe Catalysts and Transfer to Flexible Substrates, https://arxiv.org/abs/1604.07938, 2016.

[14] N. Chiodarelli, S. Masahito, Y. Kashiwagi, Y. Li, K. Arstila, O. Richard, et al., Measuring the electrical resistivity and contact resistance of vertical carbon nanotube bundles for application as interconnects, Nanotechnology (2011) 22(8) 085302, 7pp.

[15] International Technology Roadmap for Semiconductors (ITRS), Interconnect, , web: https://www.dropbox.com/sh/6xq737bg6pww9gq/AADSTNSMIs7gD9IInnI4WFHea/2013In terconnect.pdf?dl=0, 2013.

[16] M.H. van der Veen, B. Vereecke, C. Huyghebaert, D.J. Cott, M. Sugiura, Y. Kashiwagi, et al., Electrical characterization of CNT contacts with $\mathrm{Cu}$ Damascene top contact, Microelectronic Engineering (2013) 106 106-111.

[17] A.P. Graham, G.S. Duesberg, W. Hoenlein, F. Kreupl, M. Liebau, R. Martin, et al., How do carbon nanotubes fit into the semiconductor roadmap?, Applied Physics a-Materials Science \& Processing (2005) 80(6) 1141-1151. 
[18] C. Nunes Kirchner, K.H. Hallmeier, R. Szargan, T. Raschke, C. Radehaus, G. Wittstock, Evaluation of Thin Film Titanium Nitride Electrodes for Electroanalytical Applications, Electroanalysis (2007) 19(10) 1023-1031.

[19] J. Uhm, H. Jeon, TiN Diffusion Barrier Grown by Atomic Layer Deposition Method for $\mathrm{Cu}$ Metallization, Japanese Journal of Applied Physics (2001) 40(Part 1, No. 7) 4657-4660.

[20] Y.-H. Shin, Y. Shimogaki, Diffusion barrier property of TiN and TiN/Al/TiN films deposited with FMCVD for Cu interconnection in ULSI, Science and Technology of Advanced Materials (2004) 5(4) 399-405.

[21] J.V. Anguita, M. Ahmad, S. Haq, J. Allam, S.R.P. Silva, Ultra-broadband light trapping using nanotextured decoupled graphene multilayers, Sci. Adv. (2016) 2(2) 8.

[22] G. Zhong, J.H. Warner, M. Fouquet, A.W. Robertson, B. Chen, J. Robertson, Growth of Ultrahigh Density Single-Walled Carbon Nanotube Forests by Improved Catalyst Design, ACS Nano (2012) 6(4) 2893-2903.

[23] G. Zhong, R. Xie, J. Yang, J. Robertson, Single-step CVD growth of high-density carbon nanotube forests on metallic $\mathrm{Ti}$ coatings through catalyst engineering, Carbon (2014) 67(Supplement C) 680-687.

[24] C. Lu, J. Liu, Controlling the Diameter of Carbon Nanotubes in Chemical Vapor Deposition Method by Carbon Feeding, The Journal of Physical Chemistry B (2006) 110(41) 20254-20257.

[25] C. Thomsen, S. Reich, Double Resonant Raman Scattering in Graphite, Physical Review Letters (2000) 85(24) 5214-5217.

[26] W. Li, H. Zhang, C. Wang, Y. Zhang, L. Xu, K. Zhu, et al., Raman characterization of aligned carbon nanotubes produced by thermal decomposition of hydrocarbon vapor, Applied Physics Letters (1997) 70(20) 2684-2686.

[27] S. Riichiro, J. Ado, G.S.F. Antonio, D. Gene, S.D. Mildred, G. Alexander, et al., First and Second-Order Resonance Raman Process in Graphite and Single Wall Carbon Nanotubes, Japanese Journal of Applied Physics (2002) 41(7S) 4878.

[28] M. Ahmad, J.V. Anguita, V. Stolojan, J.D. Carey, S.R.P. Silva, Efficient Coupling of Optical Energy for Rapid Catalyzed Nanomaterial Growth: High-Quality Carbon Nanotube Synthesis at Low Substrate Temperatures, ACS Applied Materials \& Interfaces (2013) 5(9) 3861-3866.

[29] A.C. Ferrari, Raman spectroscopy of graphene and graphite: Disorder, electron-phonon coupling, doping and nonadiabatic effects, Solid State Communications (2007) 143(1) 47-57. [30] Y.T. Lee, N.S. Kim, J. Park, J.B. Han, Y.S. Choi, H. Ryu, et al., Temperature-dependent growth of carbon nanotubes by pyrolysis of ferrocene and acetylene in the range between 700 and 1000 degrees C, Chemical Physics Letters (2003) 372(5-6) 853-859.

[31] M.S. Shamsudin, N.A. Asli, S. Abdullah, S.Y.S. Yahya, M. Rusop, Effect of Synthesis Temperature on the Growth Iron-Filled Carbon Nanotubes as Evidenced by Structural, MicroRaman, and Thermogravimetric Analyses, Adv Cond Matter Phys (2012) (Article ID 420619) [32] C.J. Lee, J. Park, Y. Huh, J.Y. Lee, Temperature effect on the growth of carbon nanotubes using thermal chemical vapor deposition, Chemical Physics Letters (2001) 343(1-2) 33-38.

[33] J. Maultzsch, S. Reich, C. Thomsen, S. Webster, R. Czerw, D.L. Carroll, et al., Raman characterization of boron-doped multiwalled carbon nanotubes, Applied Physics Letters (2002) 81(14) 2647-2649.

[34] L.G. Bulusheva, A.V. Okotrub, I.A. Kinloch, I.P. Asanov, A.G. Kurenya, A.G. Kudashov, et al., Effect of nitrogen doping on Raman spectra of multi-walled carbon nanotubes, Physica Status Solidi (b) (2008) 245(10) 1971-1974.

[35] J.E. Sundgren, Structure and Properties of TiN Coatings, Thin Solid Films (1985) 128(12) $21-44$. 
[36] D. Yokoyama, T. Iwasaki, T. Yoshida, H. Kawarada, S. Sato, T. Hyakushima, et al., Low temperature grown carbon nanotube interconnects using inner shells by chemical mechanical polishing, Applied Physics Letters (2007) 91(26) 263101.

[37] N. Chiodarelli, Y. Li, D.J. Cott, S. Mertens, N. Peys, M. Heyns, et al., Integration and electrical characterization of carbon nanotube via interconnects, Microelectronic Engineering (2011) 88(5) 837-843.

[38] J. Li, Q. Ye, A. Cassell, H.T. Ng, R. Stevens, J. Han, et al., Bottom-up approach for carbon nanotube interconnects, Applied Physics Letters (2003) 82(15) 2491-2493.

[39] V. Gopee, O. Thomas, C. Hunt, V. Stolojan, J. Allam, S.R.P. Silva, Carbon Nanotube Interconnects Realized through Functionalization and Sintered Silver Attachment, ACS Applied Materials \& Interfaces (2016) 8(8) 5563-5570.

[40] S. Vollebregt, F.D. Tichelaar, H. Schellevis, C.I.M. Beenakker, R. Ishihara, Carbon nanotube vertical interconnects fabricated at temperatures as low as $350^{\circ} \mathrm{C}$, Carbon (2014) 71 249-256.

[41] D. Jiang, T. Wang, S. Chen, L. Ye, J. Liu, Paper-mediated controlled densification and low temperature transfer of carbon nanotube forests for electronic interconnect application, Microelectronic Engineering (2013) 103 177-180.

[42] A.C. Ferrari, D.M. Basko, Raman spectroscopy as a versatile tool for studying the properties of graphene, Nature Nanotechnology (2013) 8235.

[43] X. Li, W. Cai, J. An, S. Kim, J. Nah, D. Yang, et al., Large-Area Synthesis of High-Quality and Uniform Graphene Films on Copper Foils, Science (2009) 324(5932) 1312.

[44] A. Reina, X. Jia, J. Ho, D. Nezich, H. Son, V. Bulovic, et al., Large Area, Few-Layer Graphene Films on Arbitrary Substrates by Chemical Vapor Deposition, Nano Letters (2009) 9(1) $30-35$.

[45] J.V. Anguita, D.C. Cox, M. Ahmad, Y.Y. Tan, J. Allam, S.R.P. Silva, Highly Transmissive Carbon Nanotube Forests Grown at Low Substrate Temperature, Advanced Functional Materials (2013) 23(44) 5502-5509.

[46] G.Y. Chen, B. Jensen, V. Stolojan, S.R.P. Silva, Growth of carbon nanotubes at temperatures compatible with integrated circuit technologies, Carbon (2011) 49(1) 280-285.

[47] J. Clark, J.V. Anguita, Y. Chen, S.R.P. Silva, Optical nanostructures in 2D for widediameter and broadband beam collimation, Sci Rep (2016) 610. 